\title{
零 McGill
}

Petrides, M. and Segal, E.

The morphology and variability of the caudal rami of the superior temporal sulcus.

Submitted to and accepted by:

European Journal of Neuroscience 2012 Jul. 36(1):2035-2053 Epub 2012 Jun 18.

Rights copyright retained by the authors.

doi: $10.1111 / \mathrm{j} .1460-9568.2012 .08109 . x$

http://www.ncbi.nlm.nih.gov/pubmed/22708629

Uploaded to eScholarship@McGill.ca on 1 October 2015. All items in eScholarship@McGill.ca are protected by copyright with all rights reserved unless otherwise indicated. 
Segal E., and Petrides M. (2012). The morphology and variability of the caudal rami of the superior temporal sulcus.

Submitted to The European Journal of Neuroscience, 36 (1), 2035-2053.

This research was supported by Canadian Institutes of Health Research (CIHR) grant MOP14620 to M. Petrides. 


\begin{abstract}
The caudal branches of the superior temporal sulcus (cSTS) have been difficult to characterize because of the considerable degree of morphological variability across individuals. Leading atlases of the human brain are inconsistent with each other in terms of the number of branches identified and the nomenclature used to refer to them. Examination of the magnetic resonance images (MRIs) of 45 human brains (90 hemispheres) demonstrates three branches of the cSTS that ascend into the inferior parietal lobule: an anterior branch (cSTS1), a central branch (cSTS2) and a posterior branch (cSTS3). The cSTS1 is found immediately posterior to the ascending limb of the Sylvian fissure, followed by the cSTS2, and then the last branch, cSTS3, at the parieto-occipital junction. The temporal part of the STS joins most frequently with the cSTS2 (approximately 60\% of cases), the cSTS1 (approximately 30\% of cases) and least frequently with the cSTS3 (approximately 10\% of cases). At the temporo-occipital junction, there is another sulcus that is related to the STS, the ventral anterior occipital sulcus (AOCS-v), a sulcus that has been functionally linked to area MT/v5 in the human brain. While the STS may appear to join AOCS-v from the surface of the brain, it can be established from examination of the depth of the sulci that they are not continuous. The variability in location of each one of the cSTS branches is expressed quantitatively in the MNI standard proportional stereotaxic space.
\end{abstract}




\section{Introduction}

The morphology of the caudal part of the superior temporal sulcus (cSTS), which extends into the inferior parietal lobule (IPL), is complex in human brains. Comparative investigation (Shellshear, 1927) suggests that, from the single cSTS of lower primates (also called the superior parallel sulcus), two sulci are extruded in the human brain, the dorsal and ventral anterior occipital sulci (AOCS-d and AOCS-v). The AOCS-d remains within the IPL near the parietooccipital region, while the AOCS-v lies more ventrally at the temporooccipital junction. Between the newly extruded AOCS-d and the parallel sulcus from which it is extruded, a new fold is formed - the angular sulcus (see Figure 3.1). Thus, based on the evolutionary research of Shellshear (1927), we should expect three branches of the cSTS within the human IPL: an anterior branch behind the ascending limb of the Sylvian fissure, a posterior branch near the occipital region and a central branch running between them.

Most modern texts and atlases (e.g. Watson, 1995; Parent \& Carpenter, 1996; Mai et al., 2007; Haines, 2008; Woolsey et al., 2008; Kiernan \& Barr, 2009; Felten \& Shetty, 2010) do not discuss the sulci of the IPL in any detail, and there are discrepancies between the few that do deal with them (Ono et al., 1990; Duvernoy, 1999). For example, schematic illustrations of the brain's lateral surface in both Duvernoy (1999) and Ono et al. (1990) represent two cSTS branches within the IPL. The schematic diagram in Duvernoy (1999, p. 7) labels two branches of the cSTS as the ascending and the horizontal posterior segments, while Ono et al. (1990, Ch. 2, p. 16) identify two cSTS branches as the angular sulcus and the anterior occipital sulcus. At first, it may seem that the same two branches are represented in these two atlases but under different names; closer examination indicates otherwise. The cSTS branch labeled as ascending by Duvernoy (1999) lies immediately behind the posterior ascending ramus of the Sylvian fissure (ascSF; see Duvernoy, 1999; sagittal sections, pp. 258-259). However, in Ono et al. (1990) a similar sulcus found just behind the ascSF is treated as an infrequent configuration of the STS and called the double parallel-type termination (Ch. 10, p. 77). The angular sulcus of Ono et al. (1990) now appears to refer to the horizontal posterior segment of Duvernoy. Thus, the above two atlases refer to two cSTS branches but they do not seem to identify the same two sulci (see Table 3.1 for summary). Clearly, the morphology of the cSTS requires re-evaluation. The aim of the present study was to investigate the morphology of the cSTS branches. We provide anatomical descriptions of the cSTS branches and their variability in relation to surrounding 
sulci, as well as probability maps of their stereotaxic coordinates in the standard space of the Montreal Neurological Institute (MNI), which is the commonly used space for modern functional and structural neuroimaging studies.

\section{Materials and methods}

MRI scans of 45 brains (90 hemispheres) from the MNI International Consortium for Brain Mapping (ICBM) database were randomly selected for the present investigation. The mean age of the subjects (27 male, 18 female) was 25.2 ( \pm 5.3$)$ years (range: 18-40 years). Three of the subjects (one male, two female) were left-handed and the other 42 subjects were right-handed. The MRI scanning was performed with a 1.5-Tesla Philips Gyro scanner. First, stereotaxic registration of the MRI volumes was achieved using the average 305 linear symmetric target (Mazziotta et al., 2001). Cortical surfaces were extracted using the MNI CIVET pipeline (MacDonald et al., 2000; Kim et al., 2005; Ad-Dab’bagh et al., 2006). Registered MRI volumes were then visually examined and labelled using display, a freely available software program (MacDonald, 1996) which permits the simultaneous viewing of a brain in three 2-D sections (axial, coronal and sagittal planes) as well as the brain's 3-D surface. The software marks the location of a sulcus as the cursor is moved from voxel to voxel. When a chosen voxel is labelled in the coronal plane, for example, the voxel is also automatically labelled on the sagittal and axial planes. The voxel's labels are also updated on the 3-D surface. In this manner, when labelling sulci a thorough examination can be achieved because the sulci can be inspected both on the cortical surface and, importantly, within in the sulcal depth. This examination allows for an accurate examination of any submerged discontinuities in sulci that may appear as one continuous sulcus on the surface.

\section{Probability maps}

Probability maps were registered to and represented on a surface template in MNI ICBM 152 nonlinear space (Lyttelton et al., 2007). These probability maps provide a quantitative description of the location and variability of a sulcus at each $\mathrm{x}, \mathrm{y}, \mathrm{z}$ coordinate in MNI standard stereotaxic space. The sulcal labels that were created in the volume space for each brain were first transposed on to the individual cortical surfaces that had been registered to the MNI ICBM 152 nonlinear surface template (Lyttelton et al., 2007). As voxels were labelled within the 
cerebrospinal fluid that lies between the banks of a particular sulcus, the labelled region was first dilated to $1 \mathrm{~mm}$ to ensure enough coverage of the sulcus and its sulcal walls when it was transposed onto the registered cortical surface. The labels on the registered cortical surface vertices were then averaged together, for each hemisphere separately, and probability maps were computed by dividing the number of times that a labelled surface vertex was identified as a certain sulcus by the number of subjects examined. For example, a vertex at $\mathrm{x}, \mathrm{y}, \mathrm{z}$ MNI location with $100 \%$ probability value would mean that in every subject it was identified as part of the same sulcus. Thus, the probability values, displayed as color-coded maps, represent the likelihood that a given voxel is identified as a particular sulcus at a particular $\mathrm{x}, \mathrm{y}, \mathrm{z}$ location in MNI 152 nonlinear space (see Figure 3. 10).

To visualize the probability maps on an average cortical surface, we created an average mid-surface from the 45 subjects in this study (midpoint between the gray and white matter). An average of the registered mid-surfaces was created separately for the left hemisphere and for the right hemisphere. The probability maps of the sulci for the left hemisphere were then superimposed on the left hemisphere mid-surface average (45 hemispheres), and likewise the probability maps of the sulci of the right hemisphere were superimposed on the right hemisphere mid-surface average (45 hemispheres). In addition, we also regenerated surface averages by gender (an average asymmetric brain was comprised of 18 female left hemispheres and 18 female right hemispheres and an average asymmetric brain comprised of 27 male left hemispheres and 27 male right hemispheres (see Figures 3.11 and 3.12). Probability maps were then generated for the sulci that were identified in the brains of the female subjects and were superimposed on the average registered mid-surface female brain (see Figures 3.11A and C, and 3.12A and C); likewise, probability maps were generated for the sulci that were identified in the brains of male subjects and were superimposed on the average registered mid-surface male brain (see Figures 3.11B and D, and 3.12B and D).

\section{Sulcal identification}

In order to identify the branches of the cSTS, it is necessary to identify several nearby sulci that provide the boundaries of the region within which the four branches lie. These nearby sulci include the ascSF, the IPS, the anterior intermediate parietal sulcus (sometimes referred to as the anterior intermediate parietal sulcus of Jensen; aipsJ), the posterior intermediate parietal 
sulcus (pips), the parietal-occipital fissure (POF), the transverse occipical sulcus (TOCS), the lateral occipital sulcus (LOCS or prelunate sulcus) and the ascending limb of the inferior temporal sulcus (ALITS)/ AOCS-v (see Figure 3.2). These are defined below (Iaria \& Petrides, 2007).

The ascSF refers to a short sulcus that ascends more-or-less vertically from the most caudal aspect of the Sylvian fissure and is the axis around which the supramarginal gyrus is formed (see Figure 3.2).

The intraparietal sulcus (IPS) is the horizontally oriented sulcus that divides the parietal lobe into the superior parietal lobule and the IPL (see Figure 3.2).

The TOCS is found at the caudal extremity of the paroccipital part of the IPS (see Figure $3.2)$.

The LOCS or prelunate sulcus is a horizontal sulcus that extends anteriorly from the lunate sulcus (also called the sulcus prelunatus by Elliot Smith, 1907 and by Shellshear, 1927). The LOCS is found ventral to the TOCS (see Figure 3.2).

The aipsJ and the pips are relatively short, downwardly oriented sulci that branch off from the ventral aspect of the IPS (see Figure 3.2). The POF demarcates, mostly on the medial surface of the hemisphere, the parietal from the occipital lobe. It extends onto the lateral surface for a short distance (see Figure 3.2).

The AOCS-v is a sulcus in the posterior temporal region (at its border with the occipital lobe) that runs more-or-less vertically (see Figure 3.2). This sulcus, which is the ventral part of the AOCS (Shellshear,1927), has recently become the focus of considerable interest in functional neuroimaging studies because it is found to be related to the motion area MT (V5). It was called the ALITS in the first neuroimaging study that identified the motion region at the temporooccipital junction (Watson et al., 1993).

\section{Results}

Three sulci in the IPL were identified as related to the cSTS: an anterior branch, cSTS1, a central branch, cSTS2 and a posterior branch, cSTS3.

cSTS1 
The anterior branch, cSTS1, is a long sulcus that originated in the caudal temporal lobe and coursing within the IPL just behind the ascSF. It could be visualized in sagittal sections that demonstrated clearly that it was emerging from the temporal lobe and the temporal part of the STS. The anterior branch extended dorsally to approach the IPS in 16.7\% of hemispheres (13.3\% left, $20.0 \%$ right) but it never continued into the IPS, as could be clearly established in horizontal sections that permitted examination of the relation of the intraparietal sulcal complex and the sulci of the inferior parietal region. In these few cases, the cSTS1 maintained a superficial relationship with the IPS and clearly did not originate from it (see Figure 3.3A-F). The cSTS1 could, in some cases, be confused with another totally separate sulcus that appears in the same region close to the IPL, namely the aipsJ. In cases of ambiguity, an examination of horizontal sections established which of the two sulci originated close to the IPS. Such an examination revealed that the aipsJ was clearly related to the IPS while the anterior branch of the cSTS originated from the temporal region and, only superficially, approached the IPS in a few cases.

The anterior branch (i.e. cSTS1) approached the termination of the Sylvian fissure and made superficial contact with it when viewed on the brain's lateral surface in approximately $15.5 \%$ of hemispheres (17.8\% left, $13.3 \%$ right; see Figure 3.3G-L). The anterior branch was usually found anterior to the aipsJ; in only approximately $5.6 \%$ of hemispheres $(2.2 \%$ left, $8.9 \%$ right) did the cSTS1 appear posterior to the aipsJ. When viewed on the brain's lateral surface, in approximately 30\% of hemispheres (28.9\% left, 31.1\% right) the anterior branch could appear to join the aipsJ, but they were established to be separate entities because in horizontal sections it was clear that there was a submerged gyrus that separated the cSTS1 from the aipsJ (see Figure 3.4A-G). The anterior branch usually ran as a continuous sulcus but in approximately $8.9 \%$ of hemispheres $(6.7 \%$ left, $11.1 \%$ right) it appeared to be in short broken segments. In approximately $32.2 \%$ of hemispheres (22.2\% left, $42.2 \%$ right) the anterior temporal segment of the STS continued with the cSTS1. When viewed on the lateral surface of the brain, it could appear that the STS continued with the cSTS1 at a more frequent rate and in a more ambiguous way than what actually happened in the sulcal depths. For example, according to the lateral surface the STS could ambiguously appear to be joining with the cSTS1 along with the cSTS2, and in some cases along with the cSTS3, in an additional 13.3\% of hemispheres (8.9\% left, $17.8 \%$ right). It was therefore important to look into the sulcal depths to resolve the ambiguity about which of the cSTS branches the STS actually joined with (see Figure 3.4H-M). The MNI 
coordinates with the highest probability of being identified as the anterior branch were: for the left hemisphere, $\quad \mathrm{x} \quad=-55, \quad \mathrm{y}=$ $-50, \mathrm{z}=35$; and for the right hemisphere, $\mathrm{x}=55, \mathrm{y}=-41, \mathrm{z}=37$ (see Figure 3.10A and Table $3.2)$.

cSTS2

The central branch, cSTS2, was found between the anterior and the posterior branches. Its dorsal extent approached the aipsJ in approximately 5.6\% of hemispheres (4.4\% left, 6.7\% right; see Figure 3.5A-F) and the pips in approximately $11.1 \%$ of hemispheres $(11.1 \%$ left, $11.1 \%$ right; see Figure 3.5G-L). In approximately $12.2 \%$ of the cases (15.6\% of left hemispheres, 8.9\% of right hemispheres), the central branch extended dorsally and touched the IPS. Again, this was a superficial relation and it was clear from examining the horizontal sections that the central branch did not originate from the IPS (see Figure 3.6A-F). In approximately 58.9\% of hemispheres (62.2\% left, 55.6\% right) the temporal lobe segment of the STS joined with the central branch. When viewed on the lateral surface of the brain, it could appear that the STS continued with the cSTS2 at a more frequent rate and in a more ambiguous way than what actually happened in the sulcal depths. For example, according to the lateral surface the STS could ambiguously appear to be joining with the cSTS2 along with the cSTS1, and in some cases along with the cSTS3, in an additional $13.3 \%$ of hemispheres (4.4\% left, $22.2 \%$ right) and it was therefore important to examine the sulcal depths to resolve the ambiguity about which of the cSTS branches actually joined the temporal segments of the STS (see Figure 3.6G-L). The MNI coordinates with the highest probability of being identified as the central branch were $\mathrm{x}=-44, \mathrm{y}$ $=-58, \mathrm{z}=37$ and, for the right hemisphere, $\mathrm{x}=47, \mathrm{y}=-53, \mathrm{z}=35$ (see Figure 3.10B and Table $3.2)$.

cSTS3

The posterior branch, cSTS3, was found behind the central branch of the cSTS. There could also be a short connecting (i.e. annectant) sulcus, running horizontally between this branch and cSTS2 (see Figure 3.7A-F). This annectant sulcus was often located at the point where the cSTS3 separated from the AOCS-v, a ventrally located sulcus in the temporooccipital junction. The terminal end of the posterior branch pointed towards the caudal part of the IPS and above the TOCS. In approximately $12.2 \%$ of hemispheres (11.1\% left, $13.3 \%$ right), the cSTS3 
approached the TOCS or the IPS, but examination of horizontal sections clearly demonstrated that the cSTS3 did not originate from either the TOCS or the IPS and that it was a separate sulcus (see Figure 3.7G-L). A reliable way to identify the posterior branch is to examine its terminal end, which usually points towards the POF (see Figure 3.8A-F).

In approximately $10.0 \%$ of cases (15.6\% of left hemispheres, $4.4 \%$ of right hemispheres) the temporal lobe segment of the STS continued with the posterior branch. When viewed on the lateral surface of the brain, it could appear that the STS continued with the cSTS3 at a more frequent rate and in a more ambiguous way than what actually happened in the sulcal depths. For example, according to the lateral surface, the STS could ambiguously appear to join with the cSTS3 along with the cSTS2, and in some cases along with the cSTS1, in an additional 17.8\% of hemispheres (15.6\% left, 20.0\% right). It was therefore important to examine the sulcal depths to resolve the ambiguity about which of the cSTS branches actually joined with the temporal lobe segment of the STS (see Figure 3.8G-M). The MNI coordinates with the highest probability of being the posterior branch were for the left hemisphere, $x=-38, y=-72, z=23$ and, for the right hemisphere, $\mathrm{x}=41, \mathrm{y}=-64, \mathrm{z}=24$ (see Figure 3.10C and Table 3.2).

Ventral to the posterior branch of the cSTS were two sulci: the AOCS-v and the LOCS. The AOCS-v approached the posterior branch of the cSTS in approximately $40.0 \%$ of cases (37.8\% of left hemispheres, $42.2 \%$ of right hemispheres; see Figure 3.9A-F), and the LOCS approached the cSTS3 in approximately 33.3\% of cases (33.3\% of left hemispheres, 33.3\% of right hemispheres; see Figure 3.9G-L). When the lateral surface of the brain was examined, the AOCS-v could appear to extend to the temporal segment of the STS and to join it superficially in approximately $3.3 \%$ of cases (6.7\% of left hemispheres, $0.0 \%$ of right hemispheres). However, according to our study of the sulcal depths, the AOCS-v did not continue as a posterior continuation of the temporal segment of the STS. The AOCS-v and LOCS approached each other in approximately $24.4 \%$ of hemispheres (15.6\% left, 33.3\% right). The MNI coordinates with the highest probability of being identified as the AOCS-v were for the left hemisphere, $\mathrm{x}=$ 41, $y=-70, z=2$ and, for the right hemisphere, $x=43, y=-63, z=1$ (see Figure 3.10D and Table 3.2).

Hemispheric asymmetry 
The average probability maps of the cSTS1, cSTS2, cSTS3 and AOCS-v for all subjects were computed separately for the left and for the right hemispheres (see Figure 3.10A-D). The probability maps in the left hemisphere compared to the probability maps in right hemisphere show that, for all four of the cSTS branches, there is a posterior displacement of each cSTS branch in the left hemisphere compared to its counterpart in the right hemisphere (see the y coordinates in the left hemisphere vs. the right hemisphere in Figure 3.10A-D).

\section{Gender differences}

The average probability maps of the cSTS1, cSTS2, cSTS3 and AOCS-v were computed for female and male subjects separately. The probability map for the sulci in the female subjects was presented on the average female registered mid-surface and the probability map for the male subjects on the average male registered mid-surface (see Figure 3.11 and 3.12). There were no significant differences observed in the left hemisphere between males and females in terms of the $\mathrm{x}, \mathrm{y}, \mathrm{z}$ location of each one of the cSTS branches. In addition, in the right hemisphere, males and females did not appear to differ in terms of the $\mathrm{x}, \mathrm{y}, \mathrm{z}$ location of each one of the cSTS branches. However, both males and females showed a posterior displacement of the cSTS branches in the left hemisphere relative to the right hemisphere (see Figures 3.11 and 3.12), consistent with the overall finding of hemispheric asymmetry from the total-subject average noted above.

\section{Discussion}

The present study demonstrates that three branches of the cSTS can be identified in the IPL of the human brain: an anterior branch, cSTS1, a middle branch, cSTS2, and a posterior branch, cSTS3. When the lateral surface of the brain is examined, these branches may appear as the posterior continuations of the temporal segment of the STS that is running ventral and parallel to the Sylvian fissure. However, when the temporal segment of the STS is examined in the sulcal depth, it appears to continue into the inferior parietal region by joining with a single cSTS branch, with the other two cSTS branches emerging in close proximity. It most frequently joins with the cSTS2 (approximately 60\% of cases), then with the cSTS1 (approximately 30\% of cases) and, least frequently, with the cSTS3 (approximately 10\% of cases). In addition to the three cSTS branches that ascend into the IPL, there is another cSTS branch that is more ventrally located in the temporo-occipital junction region, called the AOCS-v. When the lateral surface of 
the brain is viewed, the dorsal point of the AOCS-v may at times extend to reach the temporal segment of the STS and to join it superficially.

The most variable in location in MNI standard space is the cSTS1 (i.e. it has the lowest percentage of overlap in voxels belonging to this sulcus), followed by the cSTS2 and the cSTS3. This suggests that of the three cSTS branches that ascend into the IPL, the cSTS1 may be the most difficult to identify. In our study, the cSTS1 could be identified in every single hemisphere that was examined, based on the strict criteria that the cSTS1 is 1) a significant sulcus found immediately posterior to the ascSF and 2) it originates from the posterior temporal lobe region. The cSTS1 can sometimes be confused with the aipsJ, another sulcus that is sometimes present on the surface of the IPL in a location similar to that of the cSTS1. In such cases, the distinction can be made based on the fact that the aipsJ originates in the IPS, in contrast to the cSTS1 which originates close to the temporal lobe segment of the STS. In some cases the cSTS1 may appear to blend with the aipsJ on the surface of the hemisphere, and it is necessary to examine the sulcal depths using the 2-D volumes (e.g. in the horizontal plane) to establish the separation of the cSTS1 from the aipsJ.

The cSTS1 is probably one of the sulci most frequently misidentified in the IPL. Ono et al. (1990, Ch. 2, p. 77) refer to a double parallel type termination of the STS occurring infrequently (12\% in right hemispheres and 4\% in left hemispheres). Inspection of their figures shows that this double parallel STS termination may represent rare cases in which the cSTS1 is clearly separate from aipsJ and also some distance in its origin from the temporal component of the STS. We observe that the cSTS2 serves as the most frequent posterior continuation of the STS (in approximately 60\% of cases). The cSTS2 may be identified by qualitative descriptive criteria, such as its relationship to surrounding sulci, and also quantitatively by its highest probable location in MNI standard stereotaxic space (see the Results section). Ono et al. (1990) identify this sulcus and refer to it, inconsistently, as the anterior occipital sulcus (Ono et al., 1990, Ch. 2, p. 10) or as the angular sulcus (Ono et al., 1990, Ch. 10, p. 78). Duvernoy (1999, p. 7) refers to it as the ascending part of the STS but, in the sagittal sections of the atlas, as the horizontal posterior part of the STS.

Relation to $M T / v 5$ 
The sulcus that we have identified as the posterior cSTS branch (cSTS3) has been referred to historically by different names. This has created some confusion in the literature and fails to highlight its relation to the STS. For example, the cSTS3 has also been called the superior (dorsal) anterior occipital sulcus (AOCS-d; Smith, 1907; Shellshear, 1927; Cunningham \& Romanes, 1981). This choice in nomenclature is most likely a reflection of the fact that the cSTS3 is a sulcus close to the border of the occipital lobe and the posterior parietal region, and the fact that the AOCS-v is a sulcus close to the border of the occipital lobe and posterior temporal region (Smith, 1907; Cunningham \& Robinson, 1931). As pointed out in the introduction, evidence from the evolutionary study of Shellshear (1927) directly suggests that the anterior occipital sulcus (both the superior and ventral divisions) is a component of the STS. The cortex that was in the cSTS in the macaque monkey has expanded and has come onto the lateral surface of the brains of higher primates, including humans.

The cSTS in lower primates is a relatively deep and complex fossa of buried sulcal elements, rather than a simple and shallow cortical fold (Saleem \& Logothetis, 2007; Paxinos et al., 2008). Electrophysiological recordings of the cortex buried within the cSTS in the macaque have revealed several functional areas that are involved in higher-order aspects of visual spatial processing and, in particular, the processing of motion. One of these motion areas was first studied in owl monkeys by Allman \& Kaas (1971) and named MT, and in macaques by Zeki (1974) and named as area V5. In both species, the motion area was found in the posterior middle temporal region that is related to the STS. In owl monkeys, the STS is a simple dimple but, in the macaque, a deep sulcus. Because MT/v5 is consistently related to the region of the STS in both owl monkeys and macaques, it would be reasonable to assume that a comparable area in the human brain would also be anatomically linked to the STS, and would be similarly found at the junction of the occipital lobe with the parietal and temporal lobes. Various studies attempting to localize MT/v5 in the human brain using functional neuroimaging have demonstrated a peak of activation related to motion processing within this region (Zeki et al., 1991; Watson et al., 1993; Dupont et al., 1994; Tootell \& Taylor, 1995; Tootell et al., 1995; DeYoe et al., 1996; Beauchamp et al., 1997; O’Craven et al., 1997; Culham et al., 1999; Dumoulin et al., 2000).

The activation related to MT / v5 in the human brain according to the neuroimaging studies is found consistently near a sulcus at the temporo-occipital junction, which has been called the 'ascending limb of the inferior temporal sulcus' (ALITS; Watson et al., 1993; 
Dumoulin et al., 2000). The sulcus labelled as ALITS, however, may refer to the posterior branch of the cSTS (cSTS3) or to the AOCS-v as we have defined them in the present study. For example, in Figure 6 in Watson et al. (1993), the activation related to MT / v5 is shown at the intersection of the base of cSTS3 and LOCS but in their Figure 7 the activity is shown at the intersection of AOCS-v and LOCS, leaving ALITS, ambiguously, to refer at once to both the cSTS3 and to the AOCS-v. The stereotaxic coordinates of MT/v5 as reported by Watson et al. (1993) (left hemisphere, $\mathrm{x}=-41, \mathrm{y}=-69, \mathrm{z}=2$; right hemisphere, $\mathrm{x}=41, \mathrm{y}=-67, \mathrm{z}=2$ ) refer to the junction of the cSTS3, AOCS-v and LOCS. When the same coordinates are entered into the probability maps of the current study (Figure 3.10), they have an approximately 62\% probability of being identified as the AOCS-v, and refer to a location just ventral to the probability map of the cSTS3 (see Figure 3.13A). If we now consider the location of MT / v5 within the buried cortex of the macaque STS, we should not expect to find MT/v5 in the human brain at the most dorsal part of cSTS3 (near the IPS) nor at the most ventral part of the AOCS-v, but rather at the intersection of these two related sulci that often blend with each other. This is exactly the location of MT/v5 according to the standard coordinates from functional neuroimaging studies.

Interestingly, a recent cytoarchitectonic examination of MT / v5 using histological sections from postmortem brains (Malikovic et al., 2007) has similarly determined that the MT/ v5 region in the human brain is located at the junction of cSTS3, LOCS and the AOCS-v, which is the same area that is identified by the neuroimaging studies. If the stereotaxic coordinates from the cytoarchitectonic study $(\mathrm{x}=-43, \mathrm{y}=-73, \mathrm{z}=10)$ are entered into the probability maps of the cSTS branches of the present study (Figure 3.10), again we arrive at the junction of the ventral part of the cSTS3 and the dorsal part of the AOCS-v (see Figure 3.13B). As previously pointed out, it is at the junction of these two cSTS branches that we would expect to find MT/v5 in the human brain. The fact that MT/v5, an area involved in motion processing is related to a cSTS branch agrees with what we know from classic lesion studies about the dorsal processing stream for spatial and motion information (Mishkin \& Ungerleider, 1982).

\section{Hemispheric asymmetry and gender differences}

Asymmetry of certain temporal lobe structures, such as the length of the Sylvian fissure and the shape of the planum temporale, has been repeatedly reported (e.g. Geschwind \& Levitsky, 1968; Wada et al., 1975; Galaburda et al., 1978; Ono et al., 1990; Steinmetz et al., 
1990; Witelson \& Kigar, 1992; Loftus et al., 1993; Habib et al., 1995; Duvernoy, 1999; Westbury et al., 1999; Toga \& Thompson, 2003; Lyttelton et al., 2009). The present results also suggest asymmetry of the cSTS branches. Probability maps based on data from all subjects were generated for each cSTS branch separately for each hemisphere. In Figure 3.10, we present the co-ordinates in MNI standard stereotaxic space that have the highest probability of being identified as the cSTS1, cSTS2, cSTS3 and AOCSv, within each hemisphere (Figure 3.10A-D). All of the cSTS branches appear to have a more posterior location in the left hemisphere than in the right hemisphere. We subsequently performed a separate analysis that enabled us to examine hemispheric differences according to gender. Figures 3.11 and 3.12 present the co-ordinates in MNI standard stereotaxic space that have the highest probability of being identified as the cSTS1, cSTS2, cSTS3 and AOCSv, within each hemisphere, for females and for males separately. We observe a posterior displacement of the sulci in the left hemisphere relative to the right hemisphere in both females and males, similar to what is observed in the total-subject average probability map. These findings are consistent with earlier reports of asymmetry in the caudal part of the STS that does not appear to be related to gender (Ono et al., 1990; Ochiai et al., 2004), although these earlier studies had examined only two of the branches of the cSTS identified in the present study.

\section{Relation of the cSTS branches to IPL cytoarchitecture}

An interesting question is the possible relationship between the cSTS branches within the IPL and various cytoarchitectonic areas. The central branch, i.e. cSTS2, clearly lies in the middle of the angular gyrus and would therefore correspond to area 39 of Brodmann (1909) or area PG of Economo and Koskinas (1925). A recent cytoarchitectonic study of the IPL in 10 post-mortem brains (Caspers et al., 2008) reported several subdivisions of the above two classical areas. As the latter study provides the co-ordinates of these cytoarchitectonic areas in MNI standard stereotaxic space, we compared these co-ordinates with those of the sulci provided by the present study. Cytoarchitectonic area PFm (x, y, z: -53, -54, 44; 55, -49, 45) appears to correspond well to the cortex immediately adjacent to cSTS1, area PGa (x, y, z: $-46,-65,43 ; 52,-59,39)$ to cSTS2 and area PGp (x, y, z: -43, -76, 37; 47, -73, 38) to cSTS3. Thus, the cSTS branches may relate to the different cytoarchitectonic areas within the IPL. 


\section{Conclusion}

In conclusion, the present study has examined the morphology of the cSTS in a large sample of human brains using MRIs. The location of these branches has been quantified in the form of probability maps which provide information about the location of each cSTS branch in the MNI standard stereotaxic space. The results from the present study indicate that there are three cSTS branches that ascend into the IPL, and another cSTS branch that is found lower in the region of the temporo-occipital junction. Studies of the functional significance of the region that lies at the junction of the parietal, temporal and occipital lobes have tended to report the locus of their findings in rather general terms, such as the parieto-occipitotemporal junction or the parieto-occipital or the parietotemporal junction. The provision of a detailed description of the morphology of the sulci that are found in this region and a quantitative presentation of their variability in MNI space, i.e. the standard stereotaxic space most often utilized by the neuroimaging research community, should allow functional and anatomical findings to be related to specific sulci rather than merely stated in terms of a general region. 


\section{References}

Ad-Dab’bagh, Y., Lyttelton, O.C., Muehlboeck, J.S., Lepage, C., Einarson, D., Mok, K., Ivanov, O., Vincent, R.D., Lerch, J., Fombonne, E. \& Evans, A.C. (2006) The CIVET imageprocessing environment: a fully automated comprehensive pipeline for anatomical neuroimaging research. In Corbetta, M. (Ed.) Proceedings of the 12th Annual Meeting of the Organization for Human Brain Mapping. Neuroimage, Florence, Italy.

Allman, J.M. \& Kaas, J.H. (1971) A representation of the visuafleld in the caudal third of the middle temporal gyrus of the owl monkey (Aotus trivirgatus). Brain Res., 31, 85-105.

Beauchamp, M.S., Cox, R. W. \& DeYoe, E.A. (1997) Graded effects of spatial and featural attention on human area MT and associated motion processing areas. J. Neurophysiol., 78, 516-520.

Brodmann, K. (1909) Vergleichende Localisationslehre der Grosshirnrinde in ihren Principien Dargestellt auf Grund des Zellenbaues. Barth, Leipzig.

Caspers, S., Eickhoff, S.B., Geyer, S., Scheperjans, F., Mohlberg, H., Zilles, K. \& Amunts, K. (2008) The human inferior parietal lobule in stereotaxic space. Brain Struct. Funct., 212, 481-495.

Culham, J.C., Dukelow, S.P., Vilis, T., Hassard, F.A., Gati, J.S., Menon, R.S. \& Goodale, M.A. (1999) Recovery of fMRI activation in motion area MT following storage of the motion after effect. J. Neurophysiol., 81, 388-393.

Cunningham, D.J. \& Robinson, A. (1931) Cunningham’s Textbook of Anatomy. Oxford University Press, London, New York.

Cunningham, D.J. \& Romanes, G.J. (1981) Cunningham’s Textbook of Anatomy. Oxford University Press, London, New York.

DeYoe, E.A., Carman, G.J., Bandettini, P., Glickman, S., Wieser, J., Cox, R., Miller, D. \& Neitz, J. (1996) Mapping striate and extrastriate visual areas in human cerebral cortex. Proc. Natl. Acad. Sci. USA, 93, 2382-2386.

Dumoulin, S.O., Bittar, R.G., Kabani, N.J., Baker, C.L. Jr, Le Goualher, G., Bruce Pike, G. \& Evans, A.C. (2000) A new anatomical landmark for reliable identification of human area V5/MT: a quantitative analysis of sulcal patterning. Cereb. Cortex, 10, 454-463. 
Dupont, P., Orban, G.A., De Bruyn, B., Verbruggen, A. \& Mortelmans, L. (1994) Many areas in the human brain respond to visual motion. J. Neurophysiol., 72, 1420-1424.

Duvernoy, H.M. (1999) The Human Brain: Surface, Three-Dimensional Sectional Anatomy with MRI, and Blood Supply. Springer, Wien, New York.

Economo, C. \& Koskinas, G.N. (1925) Die Cytoarchitektonik der Hirnrinde des erwachsenen Menschen. Julius Springer, Berlin.

Felten, D.L. \& Shetty, A.N. (2010) Netter's Atlas of Neuroscience. Saunders/ El sevier, Philadelphia, PA.

Galaburda, A.M., LeMay, M., Kemper, T.L. \& Geschwind, N. (1978) Right-left asymmetrics in the brain. Science, 199, 852-856.

Geschwind, N.\& Levitsky, W. (1968) Human brain: left-right asymmetries in temporal speech region. Science, 161, 186-187.

Habib, M., Robichon, F., Levrier, O., Khalil, R. \& Salamon, G. (1995) Diverging asymmetries of temporo-parietal cortical areas: a reappraisal of GeschwindGalaburda theory. Brain Lang., 48, 238-258.

Haines, D.E. (2008) Neuroanatomy: An Atlas of Structures, Sections, and Systems. Wolters Kluwer Health/Lippincott Williams \& Wilkins, Philadelphia, PA.

Iaria, G.\& Petrides, M. (2007) Occipital sulci of the human brain: variability and probability maps. J. Comp. Neurol., 501, 243-259.

Kiernan, J.A. \& Barr, M.L. (2009) Barr’s the Human Nervous System: An Anatomical Viewpoint. Wolters Kluwer Health/Lippincott Williams \& Wilkins, Philadelphia, PA.

Kim, J.S., Singh, V., Lee, J.K., Lerch, J., Ad-Dab’bagh, Y., MacDonald, D., Lee, J.M., Kim, S.I. \& Evans, A.C. (2005) Automated 3-D extraction and evaluation of the inner and outer cortical surfaces using a Laplacian map and partial volume effect classification. Neuroimage, 27, 210-221.

Loftus, W.C., Tramo, M.J., Thomas, C.E., Green, R.L., Nordgren, R.A. \& Gazzaniga, M.S. (1993) Three-dimensional quantitative analysis of hemispheric asymmetry in the human superior temporal region. Cereb. Cortex, 3, 348-355.

Lyttelton, O., Boucher, M., Robbins, S. \& Evans, A. (2007) An unbiased iterative group registration template for cortical surface analysis. Neuroimage, 34, 1535-1544. 
Lyttelton, O.C., Karama, S., Ad-Dab’bagh, Y., Zatorre, R.J., Carbonell, F., Worsley,K. \& Evans, A.C.(2009) Positional and surface area asymmetry of the human cerebral cortex. Neuroimage, 46, 895-903.

MacDonald, D. (1996) Program for Display and Segmentation of Surfaces and Volumes. McConnell Brain Imaging Center, Montreal Neurological Institute, Montreal, QC, Canada (software available from http://www.bic.mni.mcgill.ca).

MacDonald, D., Kabani, N., Avis, D. \& Evans, A.C. (2000) Automated 3-D extraction of inner and outer surfaces of cerebral cortex from MRI. Neuroimage, 12, 340-356.

Mai, J.K., Paxinos, G.\& Voss, T. (2007) Atlas of the Human Brain. Elsevier Academic Press, London.

Malikovic, A., Amunts, K., Schleicher, A., Mohlberg, H., Eickhoff, S.B., Wilms, M., PalomeroGallagher, N., Armstrong, E. \& Zilles, K. (2007) Cytoarchitectonic analysis of the human extrastriate cortex in the region of V5/MT+: a probabilistic, ster eotaxic map of area hOc5. Cereb. Cortex, 17, 562-574.

Mazziotta, J., Toga, A., Evans, A., Fox, P., Lancaster, J., Zilles, K., Woods, R., Paus, T., Simpson, G., Pike, B., Holmes, C., Collins, L., Thompson, P., MacDonald, D., Iacoboni, M., Schormann, T., Amunts, K., Palomero-Gallagher, N., Geyer, S., Parsons, L., Narr, K., Kabani, N., Le Goualher, G., Boomsma, D., Cannon, T., Kawashima, R. \& Mazoyer, B. (2001) A probabilistic atlas and reference system for the human brain: International Consortium for Brain Mapping (ICBM). Philos. Trans. R. Soc. Lond. B Biol. Sci., 356, 1293-1322.

Mishkin, M. \& Ungerleider, L.G. (1982) Contribution of striate inputs to the visuospatial functions of parieto-preoccipital cortex in monkeys. Behav. Brain Res., 6, 57-77.

Ochiai, T., Grimault, S., Scavarda, D., Roch, G., Hori, T., Riviere, D., Mangin, J.F. \& Regis, J. (2004) Sulcal pattern and morphology of the superior temporal sulcus. Neuroimage, 22, 706-719.

O’Craven, K.M., Rosen, B.R., Kwong, K.K., Treisman, A. \& Savoy, R.L. (1997) Voluntary attention modulates fMRI activity in human MT-MST. Neuron, 18, 591-598.

Ono, M., Kubik, S.\& Abernathey, C.D. (1990) Atlas of the Cerebral Sulci. G. Thieme Verlag, Thieme Medical Publishers, Inc., New York. 
Parent, A. \& Carpenter, M.B. (1996) Carpenter’s human neuroanatomy. 9th Edn. Williams \& Wilkins, Baltimore.

Paxinos, G., Huang, X.F., Petrides, M. \& Toga, A.W. (2008) The Rhesus Monkey Brain in Stereotaxic Coordinates. Academic Press, London.

Saleem, K.S. \& Logothetis, N. (2007) A Combined MRI and Histology Atlas of the Rhesus Monkey Brain in Stereotaxic Coordinates. Academic, London, Burlington, MA.

Shellshear, J.L. (1927) The evolution of the parallel sulcus. J. Anat., 61, 267-278.

Smith, G.E. (1907) A new topographical survey of the human cerebral cortex, being an account of the distribution of the anatomically distinct cortical areas and their relationship to the cerebral sulci. J. Anat. Physiol., 41, 237-254.

Steinmetz, H., Rademacher, J., Jancke, L., Huang, Y.X., Thron,A. \& Zilles,K. (1990) Total surface of temporoparietal intrasylvian cortex: diverging left-right asymmetries. Brain Lang., 39, 357-372.

Toga, A.W. \& Thompson, P.M. (2003) Mapping brain asymmetry. Nat. Rev. Neurosci., 4, 3748.

Tootell, R.B. \& Taylor, J.B. (1995) Anatomical evidence for MT and additional cortical visual areas in humans. Cereb. Cortex, 5, 39-55.

Tootell, R.B., Reppas, J.B., Kwong, K.K., Malach, R., Born, R.T., Brady, T.J., Rosen, B.R. \& Belliveau, J.W. (1995) Functional analysis of human MT and related visual cortical areas using magnetic resonance imaging. J. Neurosci., 15, 3215-3230.

Wada, J.A., Clarke, R. \& Hamm, A. (1975) Cerebral hemispheric asymmetry in humans. Cortical speech zones in 100 adults and 100 infant brains. Arch. Neurol., 32, 239-246.

Watson, C. (1995) Basic Human Neuroanatomy: An Introductory Atlas. Little, Brown, Boston, MA.

Watson, J.D., Myers, R., Frackowiak, R.S., Hajnal, J.V., Woods, R.P., Mazziotta, J.C., Shipp, S. \& Zeki, S. (1993) Area V5 of the human brain: evidence from a combined study using positron emission tomography and magnetic resonance imaging. Cereb. Cortex, 3, 7994.

Westbury, C.F., Zatorre, R.J. \& Evans, A.C. (1999) Quantifying variability in the planum temporale: a probability map. Cereb. Cortex, 9, 392-405. 
Witelson, S.F. \& Kigar, D.L. (1992) Sylvian fissure morphology and asymmetry in men and women: bilateral differences in relation to handedness in men. J. Comp. Neurol., 323, $326-340$.

Woolsey, T.A., Hanaway,J. \& Gado, M.H. (2008) The Brain Atlas: A Visual Guide to the Human Central Nervous System. Wiley, Hoboken, NJ.

Zeki, S.M. (1974) Functional organization of a visual area in the posterior bank of the superior temporal sulcus of the rhesus monkey. J. Physiol., 236, 549- 573.

Zeki, S., Watson, J.D., Lueck, C.J., Friston, K.J., Kennard, C.\& Frackowiak, R.S. (1991) A direct demonstration of functional specialization in human visual cortex. J. Neurosci., 11, 641-649.

\section{Acknowledgements}

We wish to thank Dr. Claude Lepage for his help with generating the probability maps and with creating the average mid-surfaces. We also thank Dr. Oliver Lyttelton for suggestions in constructing the probability maps and Sebastian Muehlboeck for technical support. This research was supported by the Canadian Institutes of Health Research Grant MOP-14620 and grant CDR104684.

\footnotetext{
Abbreviations

aipsJ, anterior intermediate parietal sulcus of Jensen; ALITS, ascending limb of the inferior temporal sulcus; AOCS-v, anterior occipital sulcus, ventral division; ascSF, ascending limb of the Sylvian fissure; CS, central sulcus; cSTS, caudal aspect of the STS; cSTS1, anterior branch of the cSTS; cSTS2, central branch of the cSTS; cSTS3, posterior branch of the cSTS; ICBM, International Consortium for Brain Mapping; IPL, inferior parietal lobule; IPS, intraparietal sulcus; LOCS, lateral occipital sulcus; MNI, Montreal Neurological Institute; PCS, postcentral sulcus; PFm, supramarginal cytoarchitectonic area PF, magnocellular part; PGa, angular gyrus cytoarchitectonic area PG, anterior part; PGp, angular gyrus cytoarchitectonic area PG, posterior part; pips, posterior intermediate parietal sulcus; POF, parietal-occipital fissure; STS, superior temporal sulcus; TOCS, transverse occipital sulcus.
} 


\section{Tables}

Table 3.1. List of cSTS branches according to different authors and atlases

\begin{tabular}{|c|c|c|c|c|c|c|}
\hline $\begin{array}{l}\text { Segal \& } \\
\text { Petrides } \\
\text { (2012) }\end{array}$ & $\begin{array}{l}\text { Economo } \\
\text { (1925) }\end{array}$ & $\begin{array}{l}\text { Shellshear } \\
\text { (1927) }\end{array}$ & $\begin{array}{l}\text { Cunningham's } \\
\text { Textbook of } \\
\text { Anatomy, 6th } \\
\text { edition } \\
\text { (1931), pg } 670\end{array}$ & $\begin{array}{l}\text { G.E. Smith } \\
\text { (1907), } \\
\text { Figure } 2\end{array}$ & $\begin{array}{l}\text { Ono et al. } \\
\text { (1990) }\end{array}$ & $\begin{array}{l}\text { Duvernoy } \\
\text { (1999) }\end{array}$ \\
\hline $\begin{array}{l}\text { Anterior } \\
\text { Branch } \\
\text { (cSTS1) }\end{array}$ & t1' & $\begin{array}{l}\text { Sulcus } \\
\text { parallelis } \\
\text { superior }\end{array}$ & Not present & Not present & $\begin{array}{l}\text { Angular } \\
\text { Sulcus in Ch. } \\
2 \text { (p. 10); } \\
\text { referred to as } \\
\text { "double } \\
\text { parallel" type } \\
\text { in Ch. } 10 \text { (p. } \\
77) .\end{array}$ & $\begin{array}{l}\text { Not present } \\
\text { on p. 7; } \\
\text { Superior } \\
\text { Temporal } \\
\text { Sulcus } \\
\text { ascending } \\
\text { part in } \\
\text { Continuous } \\
\text { Sagittal } \\
\text { Sections }\end{array}$ \\
\hline $\begin{array}{l}\text { Central } \\
\text { Branch } \\
\text { (cSTS2) }\end{array}$ & t1" & $\begin{array}{l}\text { Sulcus } \\
\text { Angularis }\end{array}$ & $\begin{array}{l}\text { Sulcus } \\
\text { Angularis }\end{array}$ & $\begin{array}{l}\text { Sulcus } \\
\text { Angularis }\end{array}$ & $\begin{array}{l}\text { Anterior } \\
\text { Occipital } \\
\text { Sulcus in Ch. } \\
2 \text { (p. 10); } \\
\text { referred to as } \\
\text { Angular } \\
\text { Sulcus in Ch. } \\
10 \text { (p. 78). }\end{array}$ & $\begin{array}{l}\text { Superior } \\
\text { Temporal } \\
\text { Sulcus } \\
\text { ascending } \\
\text { part on p. 7; } \\
\text { Superior } \\
\text { Temporal } \\
\text { Sulcus } \\
\text { horizontal } \\
\text { posterior } \\
\text { segment in } \\
\text { Continuous } \\
\text { Sagittal } \\
\text { Sections } \\
\end{array}$ \\
\hline $\begin{array}{l}\text { Posterior } \\
\text { Branch } \\
\text { (cSTS3) }\end{array}$ & t2' & $\begin{array}{l}\text { Sulcus } \\
\text { Occipitalis } \\
\text { Anterior, } \\
\text { superior }\end{array}$ & $\begin{array}{l}\text { Sulcus } \\
\text { Occipitalis } \\
\text { Anterior }\end{array}$ & $\begin{array}{l}\text { Sulcus } \\
\text { Occipitalis } \\
\text { Anterior }\end{array}$ & $\begin{array}{l}\text { Inferior } \\
\text { Temporal } \\
\text { Sulcus in Ch. } \\
2 \text { (p. 10); } \\
\text { referred to as } \\
\text { Anterior } \\
\text { Occipital } \\
\text { Sulcus in Ch. } \\
10 \text { (p. 78) }\end{array}$ & $\begin{array}{l}\text { Superior } \\
\text { Temporal } \\
\text { Sulcus } \\
\text { horizontal } \\
\text { posterior } \\
\text { segment on } \\
\text { p. 7; LOCS } \\
\text { in } \\
\text { Continuous } \\
\text { Sagittal } \\
\text { Sections } \\
\end{array}$ \\
\hline AOCS-v & $\begin{array}{l}\text { t2"/Sulcus } \\
\text { Occipitals } \\
\text { Anterior }\end{array}$ & $\begin{array}{l}\text { Sulcus } \\
\text { Occipitalis } \\
\text { Anterior, } \\
\text { inferior } \\
\end{array}$ & Not present & $\begin{array}{l}\text { Sulcus } \\
\text { Occipitalis } \\
\text { Inferior }\end{array}$ & $\begin{array}{l}\text { Inferior } \\
\text { temporal } \\
\text { sulcus in Ch } \\
10 \text { (p. } 79 \text { ) }\end{array}$ & \\
\hline
\end{tabular}


Table 3.2. Coordinates of each cSTS branch in MNI standard stereotaxic space with the highest probability value.

\begin{tabular}{|lcccc|}
\hline Left Hemisphere & $\boldsymbol{X}$ & $\boldsymbol{Y}$ & $\mathbf{Z}$ & Probability \\
\hline $\begin{array}{l}\text { Anterior branch } \\
\text { (cSTS1) }\end{array}$ & -55 & -50 & 35 & $53 \%$ \\
\hline $\begin{array}{l}\text { Central branch } \\
\text { (cSTS2) }\end{array}$ & -44 & -58 & 37 & $60 \%$ \\
\hline $\begin{array}{l}\text { Posterior branch } \\
\text { (cSTS3) }\end{array}$ & -38 & -72 & 23 & $67 \%$ \\
\hline $\begin{array}{l}\text { AOCS- } v \\
\text { Right Hemisphere }\end{array}$ & $\mathbf{X}$ & -70 & 2 & $\mathbf{Z}$ \\
\hline $\begin{array}{l}\text { Anterior branch } \\
\text { (cSTS1) }\end{array}$ & 55 & $\boldsymbol{Y}$ & $37 \%$ & $51 \%$ \\
\hline $\begin{array}{l}\text { Central branch } \\
\text { (cSTS2) }\end{array}$ & 47 & -41 & 35 & $56 \%$ \\
\hline $\begin{array}{l}\text { Posterior branch } \\
\text { (cSTS3) }\end{array}$ & 41 & -63 & 24 & $76 \%$ \\
\hline AOCS-v & 43 & -64 & 1 & $69 \%$ \\
\hline
\end{tabular}

All peaks are reported in MNI standard stereotaxic space. Probability refers to the likelihood that the voxel ( $\mathrm{x}, \mathrm{y}, \mathrm{z}$ coordinates) is labelled as a particular sulcus. For example, in the left hemisphere the voxel with the highest likelihood (at a 53\% probable chance) of being identified as cSTS1 is at MNI coordinates -55, -50, 35 whereas in the right hemisphere the voxel with the highest likelihood (at a 51\% probable chance) of being identified as cSTS1 is at MNI coordinates $55,-41,37$, suggesting there is slightly less variability in the location of cSTS1 in the left hemisphere than in the right hemisphere. 


\section{Figures}

a)

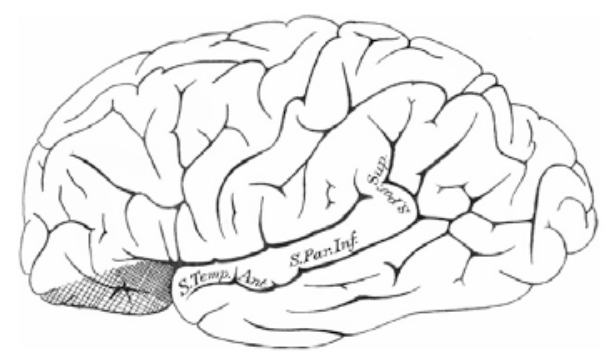

b)
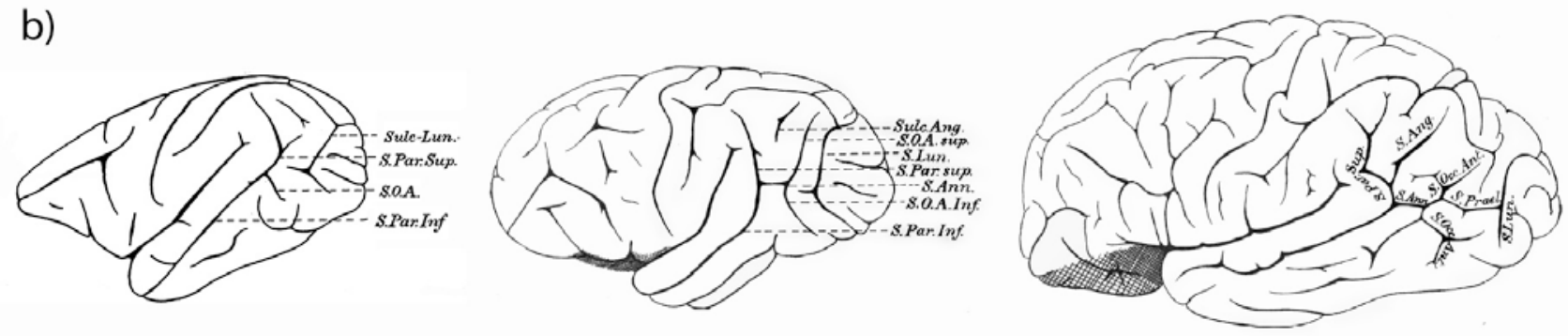

Figure 3.1. (A) The three segments of the STS in the antero-posterior direction, adapted from Figure 10 in Shellshear (1927). The STS can be divided into an anterior temporal segment (anterior temporal sulcus) and a posterior temporal segment (the inferior parallel sulcus). One of its posterior parietal segments is here labelled the superior parallel sulcus. S. Temp. Ant., anterior temporal sulcus; S. Par. Inf., inferior parallel sulcus; S. Par. Sup., superior parallel sulcus. (B) The evolutionary expansion of the cSTS branches illustrated by a comparison of the brains of the gibbon, orangutan and the human (from left to right), adapted from Figures 5, 7 and 10 in Shellshear (1927). From the posterior wall of the superior parallel sulcus in lower primates (such as the gibbon), the anterior occipital sulcus (AOCS), here labelled as SOA, is extruded in the brains of higher primates (such as the orangutan and human) where it is divided into a dorsal and a ventral branch. The dorsal AOCS stays within the inferior parietal lobule while the ventral AOCS is located in the posterior temporo-occipital cortex. In the orangutan, the angular sulcus has made its appearance between the superior parallel sulcus and the dorsal AOCS, and in the human brain the angular sulcus is even more prominent. S. Ang., sulcus angularis; S. Ann., 
annectant sulcus; S. Lun., lunate sulcus; SOA and S.Occ. Ant., anterior occipital sulcus; S.O.A. sup, superior or dorsal part of the anterior occipital sulcus; S.O.A. inf, inferior or ventral part of the anterior occipital sulcus; S. Par. Inf., inferior parallel sulcus; S. Par. Sup., superior parallel sulcus; S. Prael., prelunate sulcus; Sulc. Ang., angular sulcus. 


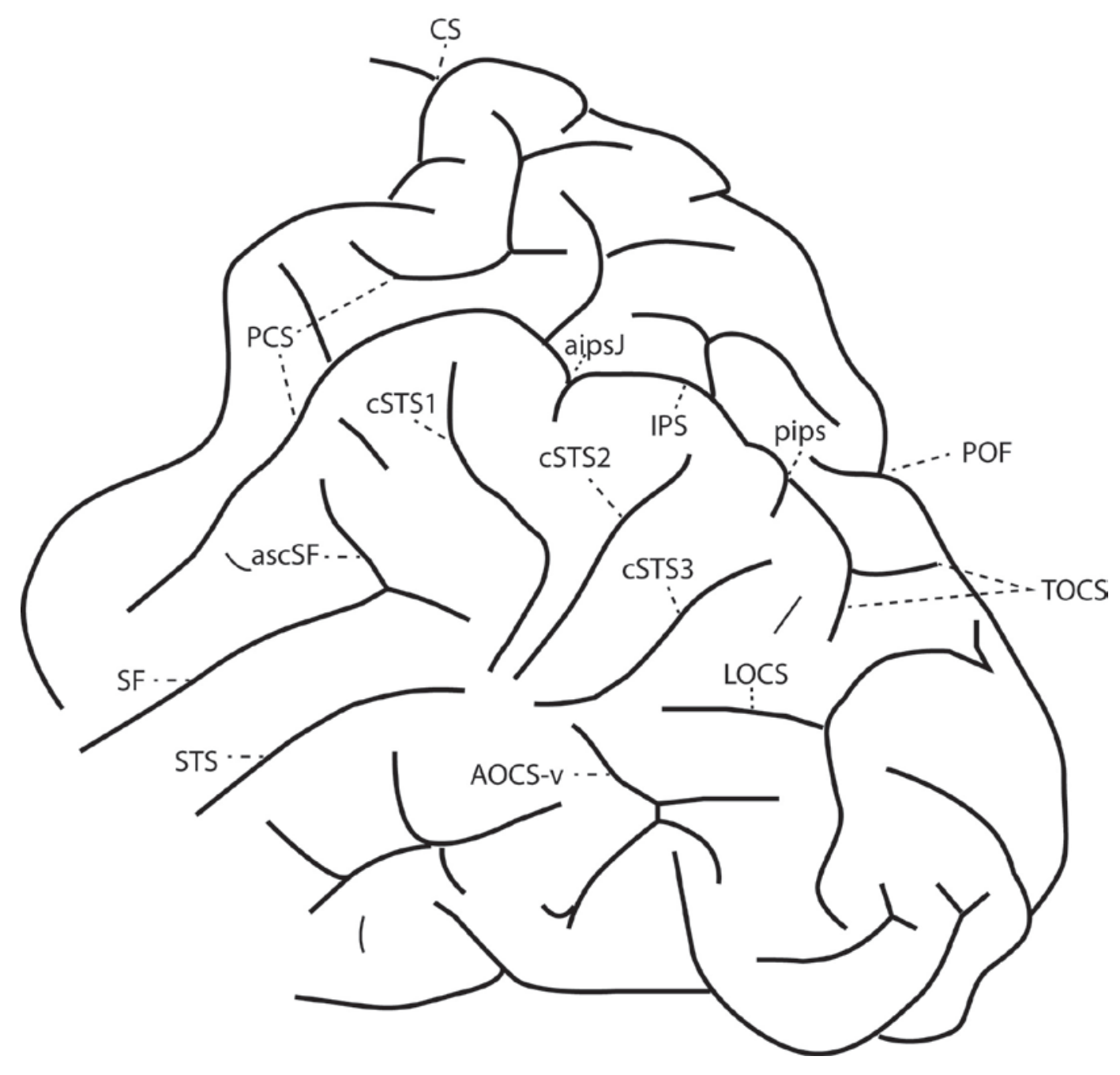

Figure 3.2. A schematic illustration of the posterior part of the lateral surface of the brain depicting the sulci relevant as landmarks for identifying the cSTS branches. aipsJ, anterior intermediate parietal sulcus of Jensen; AOCS-v, ventral division of the anterior occipital sulcus; ascSF, ascending limb of the Sylvian fissure; CS, central sulcus; cSTS1, anterior branch of the caudal STS; cSTS2, central branch of the caudal STS; cSTS3, posterior branch of the caudal STS; IPS, intraparietal sulcus; LOCS, lateral occipital sulcus; PCS, post-central sulcus; pips, posterior intermediate parietal sulcus; POF, parieto-occipital fissure; SF, Sylvian fissure; STS, superior temporal sulcus; TOCS, transverse occipital sulcus. 

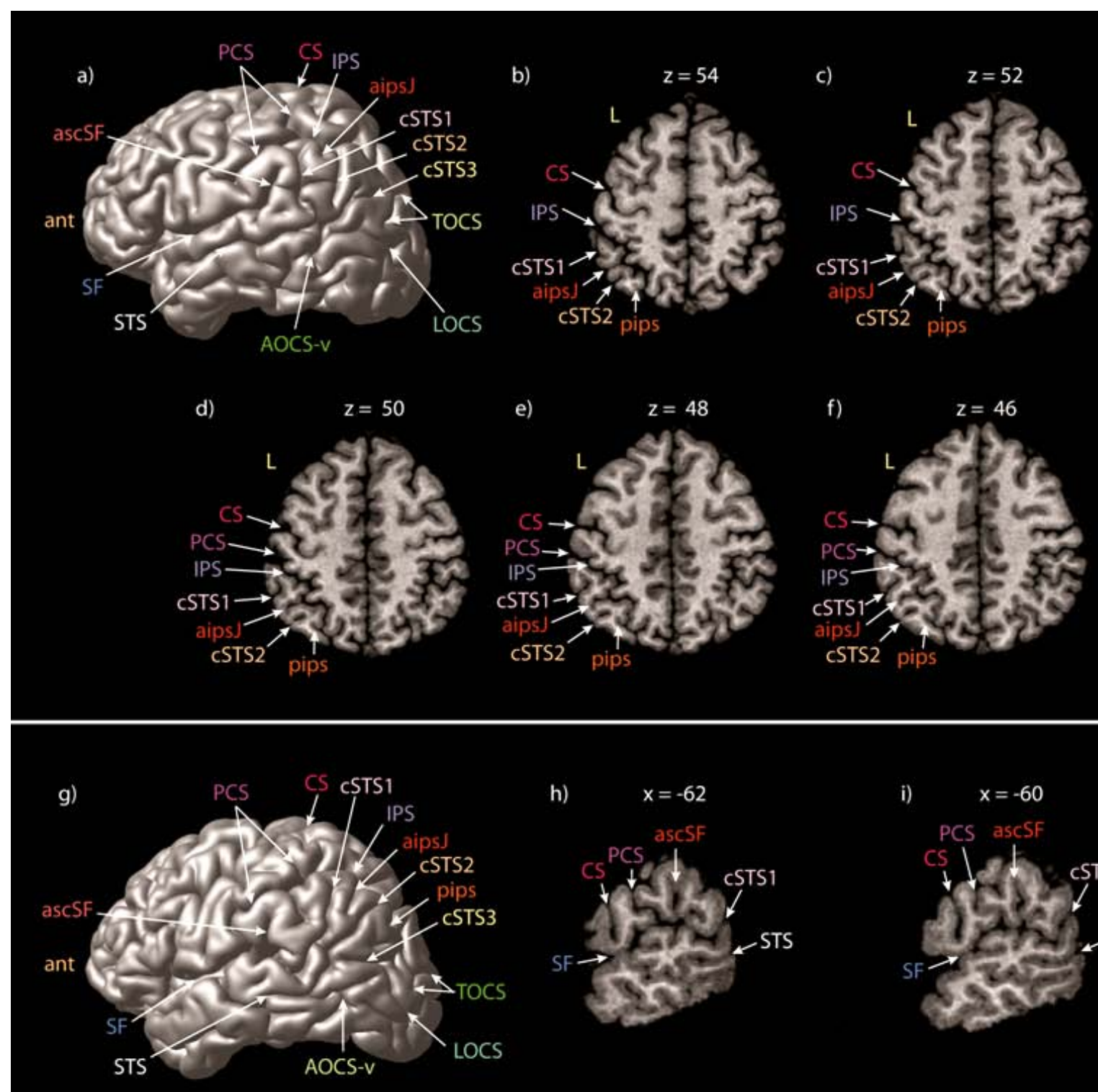

h) $\quad x=-62$

i) $x=-60$
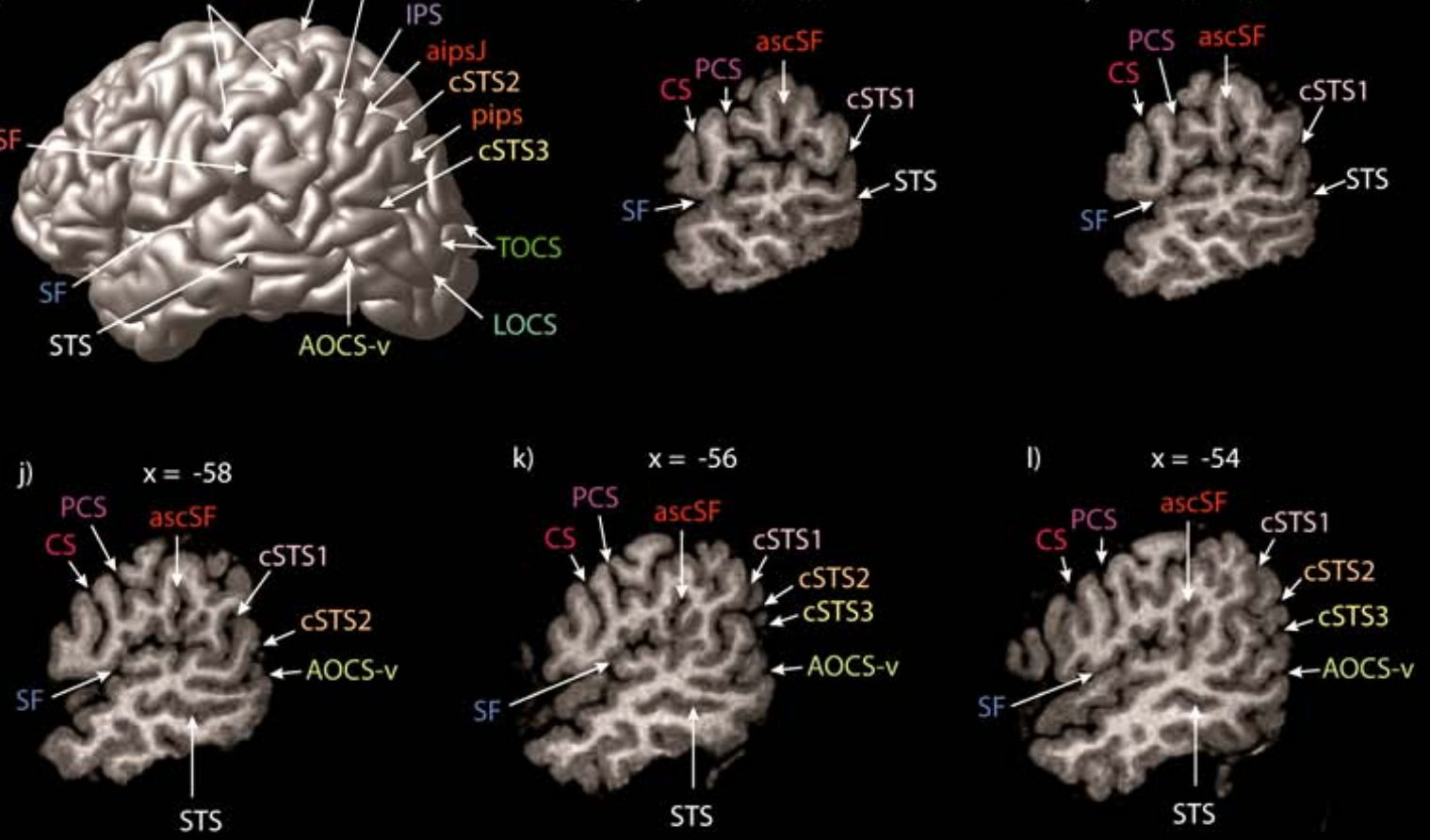
Figure 3.3. (A-F) The cSTS1 may approach the IPS as observed on (A) the 3-D lateral surface view, but (B-F) horizontal sections show that the cSTS1 is in fact a sulcus that is separate from the IPS and that does not originate within the IPS. (G-L) The cSTS1 approaches the posterior aspect of the Sylvian fissure on $(\mathrm{G})$ the 3-D lateral surface view, but (H-L) sagittal sections show that the cSTS1 is an independent sulcus, separate from the Sylvian fissure. ant, anterior; L, left hemisphere; SF, Sylvian fissure; other abbreviations in Figure 3.2 and main list. 


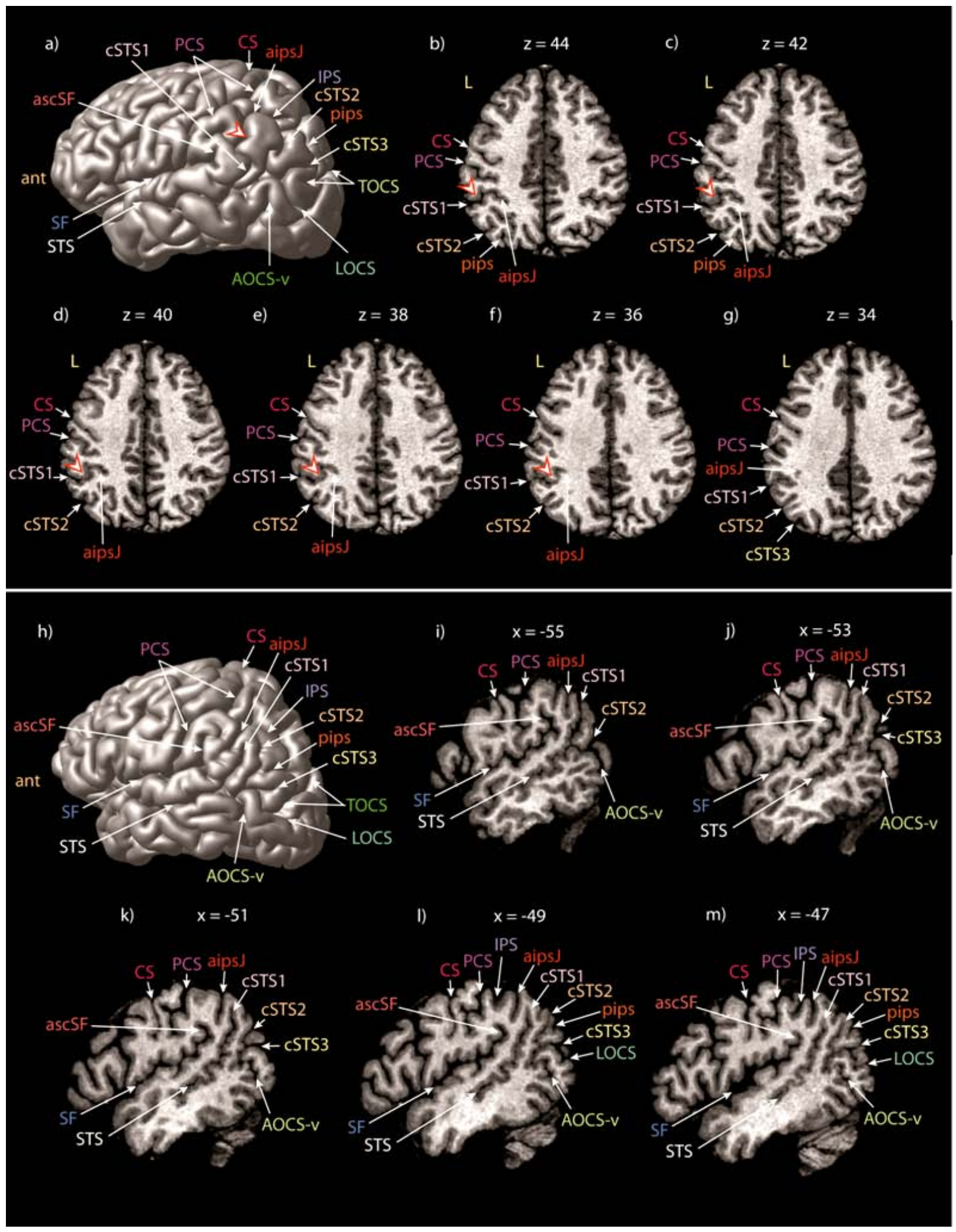


Figure 3.4. (A-G) The cSTS1 may join the aipsJ on (A) the 3-D lateral surface view but the two sulci are distinct, as can be observed in horizontal sections (B-G). The arrowhead indicates the location of the submerged gyrus that separates cSTS1 from aipsJ. (H-M) The temporal lobe segment of the STS may appear to join posteriorly with the cSTS2 and the cSTS1, according to (h) the 3-D lateral surface view, but (I-M) the sagittal sections show that, in this case in the sulcal depth, the temporal lobe segment of the STS does not join the cSTS2 but instead joins with the cSTS1, thus highlighting the importance of examining the sulcal depths and going beyond the lateral surface view. ant, anterior; L, left hemisphere; SF, Sylvian fissure; other abbreviations in Figure 3.2 and main list. 


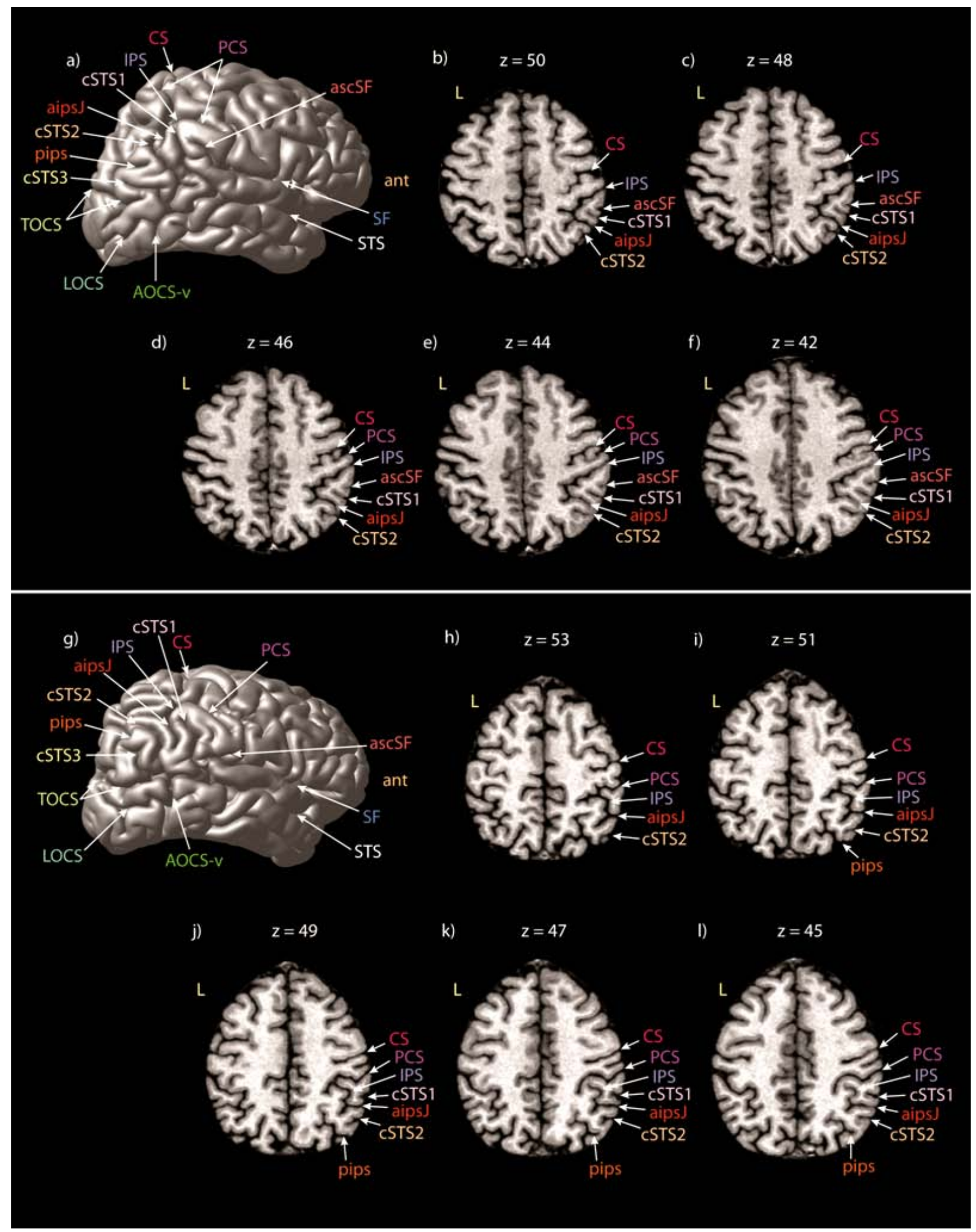


Figure 3.5. (A-F) The cSTS2 may extend dorsally to reach the aipsJ as observed in (A) the 3-D lateral surface view, but (B-F) examination of the horizontal sections demonstrate that the cSTS2 is separate from the aipsJ which originates in the IPS. (G-L) cSTS2 may extend dorsally to reach the pips $(\mathrm{G})$ but $(\mathrm{H}-\mathrm{L})$ the horizontal sections show that it is a separate sulcus and any contact with the pips is superficial. ant, anterior; L, left hemisphere; SF, Sylvifisssure; other abbreviations in Figure 3.2 and main list. 


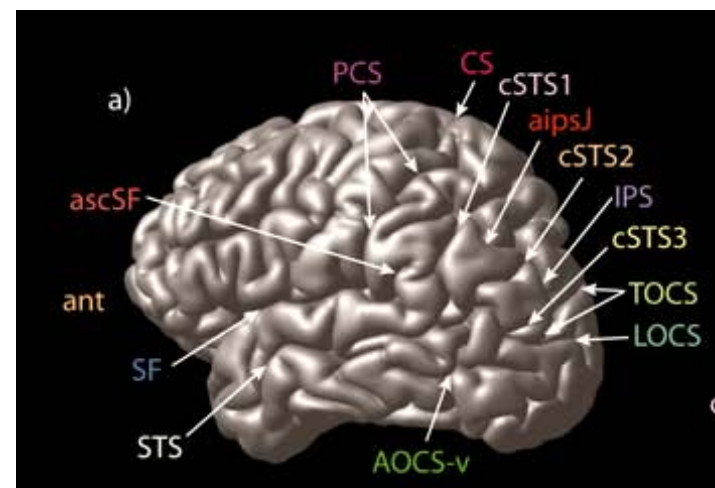
b)

$z=43$

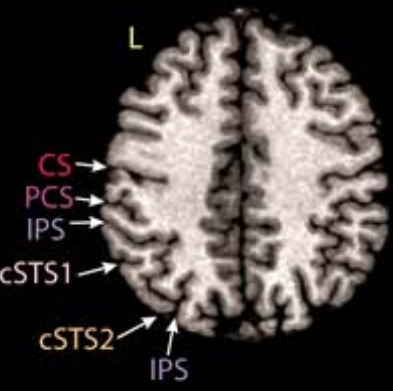

e)

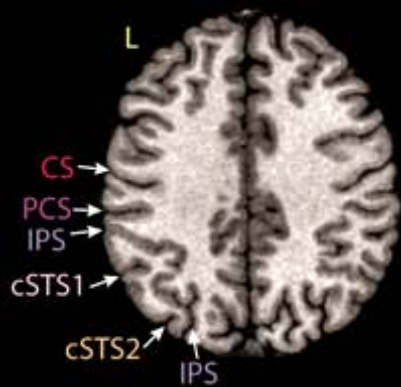

c)

$z=41$

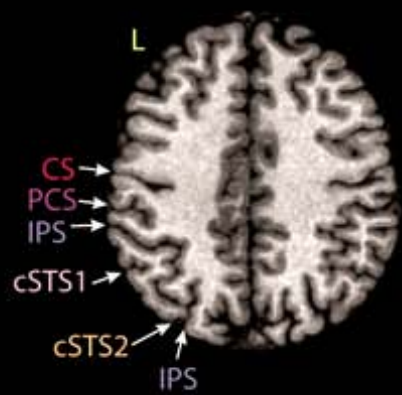

f)

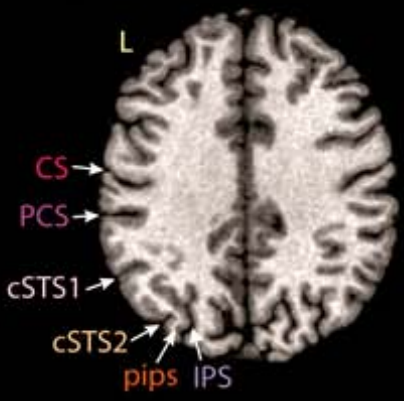

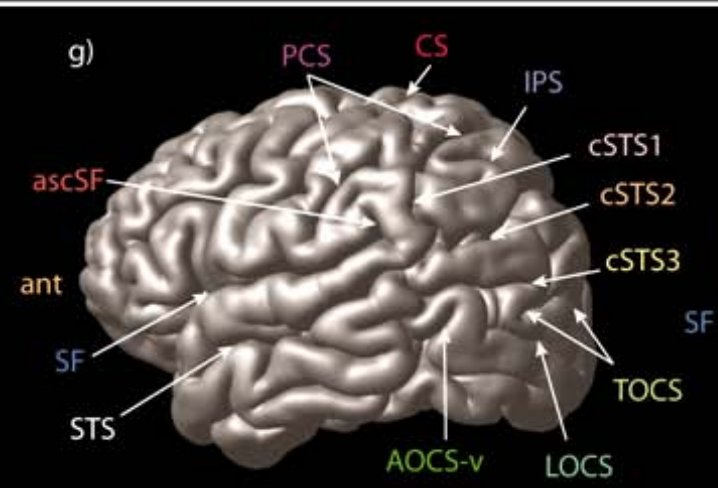

h) $x=-58$

i)

$x=-56$
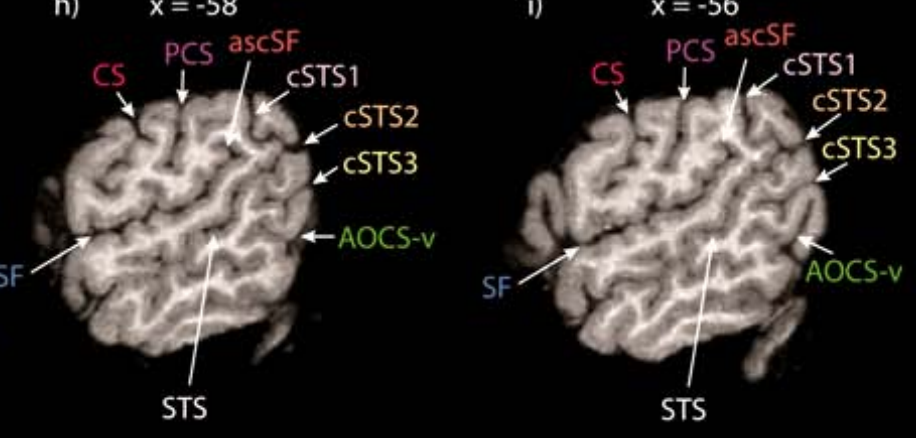

j)

k) $\quad x=-52$
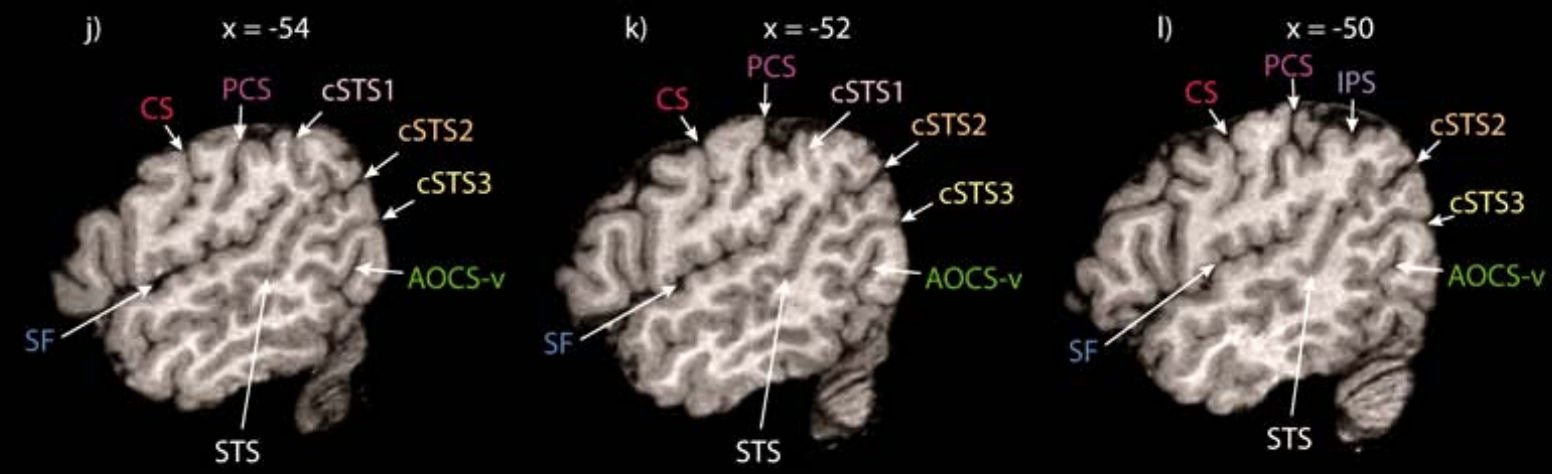
Figure 3.6. (A-F) The cSTS2 may reach dorsally and make contact with the IPS as observed from (A) the lateral surface of the brain, but (B-F) horizontal sections clearly show the separation between the cSTS2 and the IPS. (G-L) The temporal lobe segment of the STS appears continuous with cSTS1 along with the cSTS2 when viewed in $(\mathrm{G})$ the 3-D lateral surface view of the brain, but (H-L) examination of the sagittal sections shows that in this case, when the sulcal depths are examined, the temporal lobe segment of the STS in fact does not join with the cSTS1 but instead joins with cSTS2, thus highlighting the importance of examining the sulcal depths and going beyond the lateral surface view. ant, anterior; L, left hemisphere; SF, Sylvian fissure; other abbreviations in Figure 3.2 and main list. 
a)

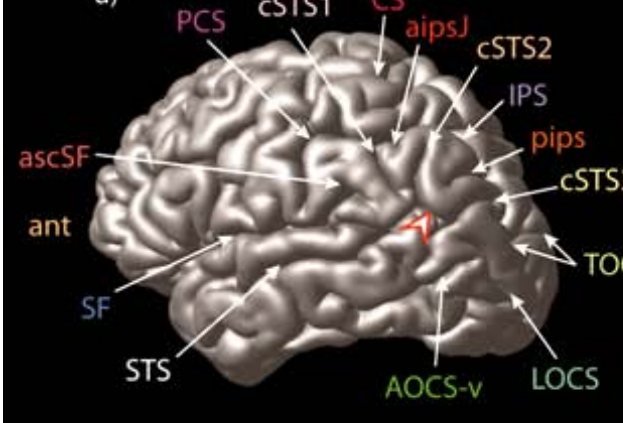

d)

$x=-46$

CS PCS IPS aipsJ

R

asCSF 2 S

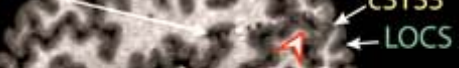

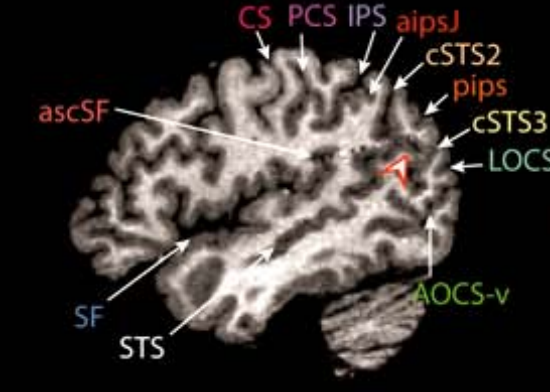

b)

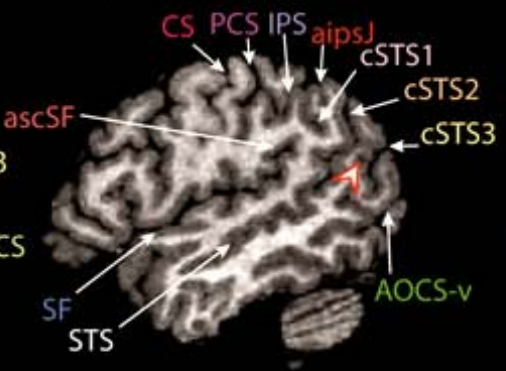

e)

$x=-44$ c)

$x=-48$

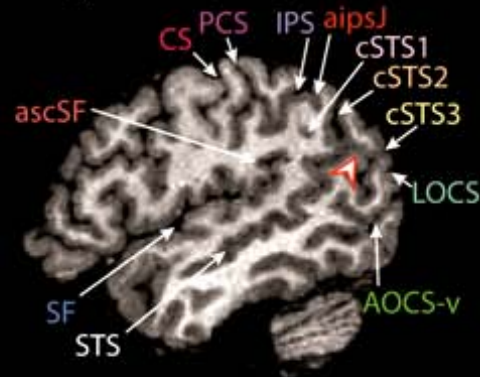

f) $x=-42$ C. C. QRN Qif
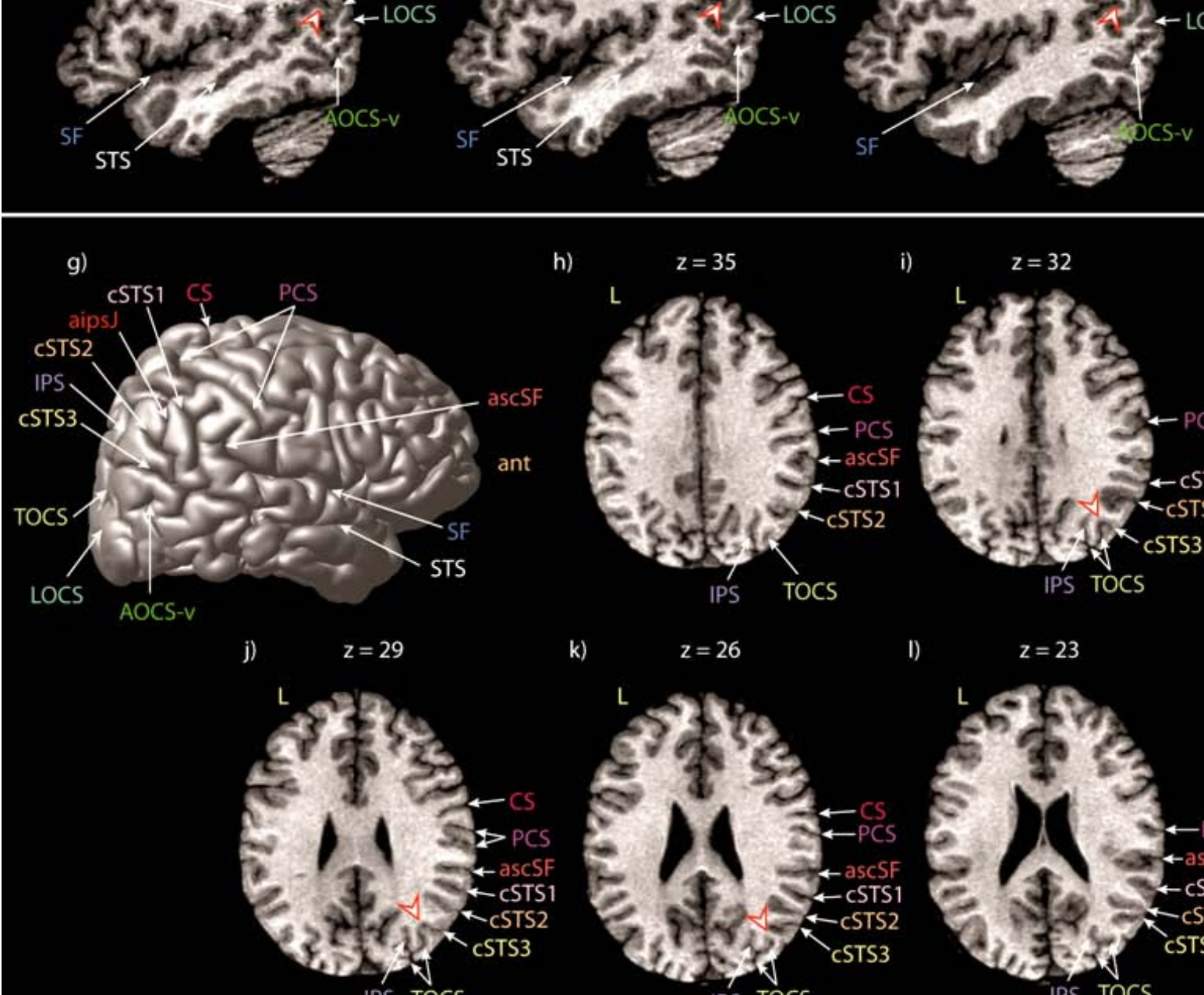

h)

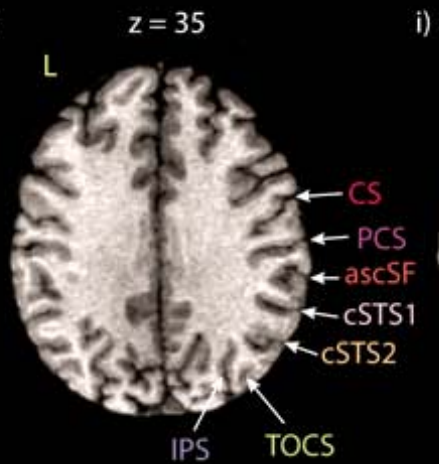

k)

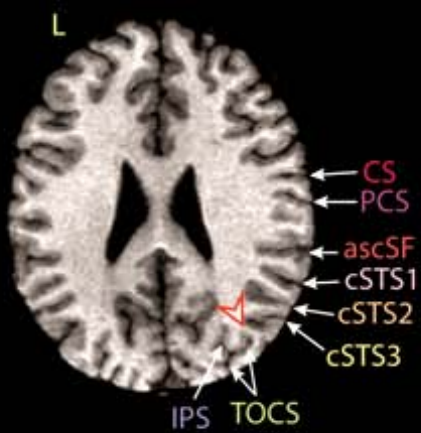

i)

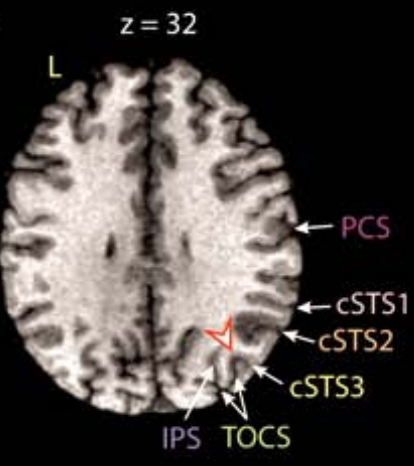

I)

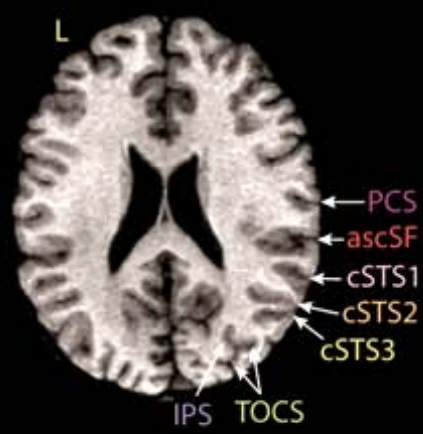


Figure 3.7. (A-F) An annectant sulcus may run between the cSTS2 and cSTS3. In some cases, the annectant sulcus can be observed from (A) the 3-D lateral surface view of the brain, but (BF) most often it is buried in the sulcal depth and can be seen in sagittal sections. An arrowhead indicates the location of the annectant sulcus. $(G-L)$ The dorsal extent of the cSTS3 may reach adjacent sulci, such as the TOCS at the ventral extent of the IPS, and appear to make contact with it, according to (G) the 3-D lateral surface view. (H-L) The horizontal sections clearly show that the cSTS3 does not originate from either the TOCS or the IPS and any contact with the TOCS or IPS is superficial. An arrowhead indicates the location of a submerged gyrus that separates the cSTS3 from the IPS/ TOCS junc tion. ant, anterior; L, left hemisphere; SF, Sylvian fissure; other abbreviations in Figure 3.2 and main list. 


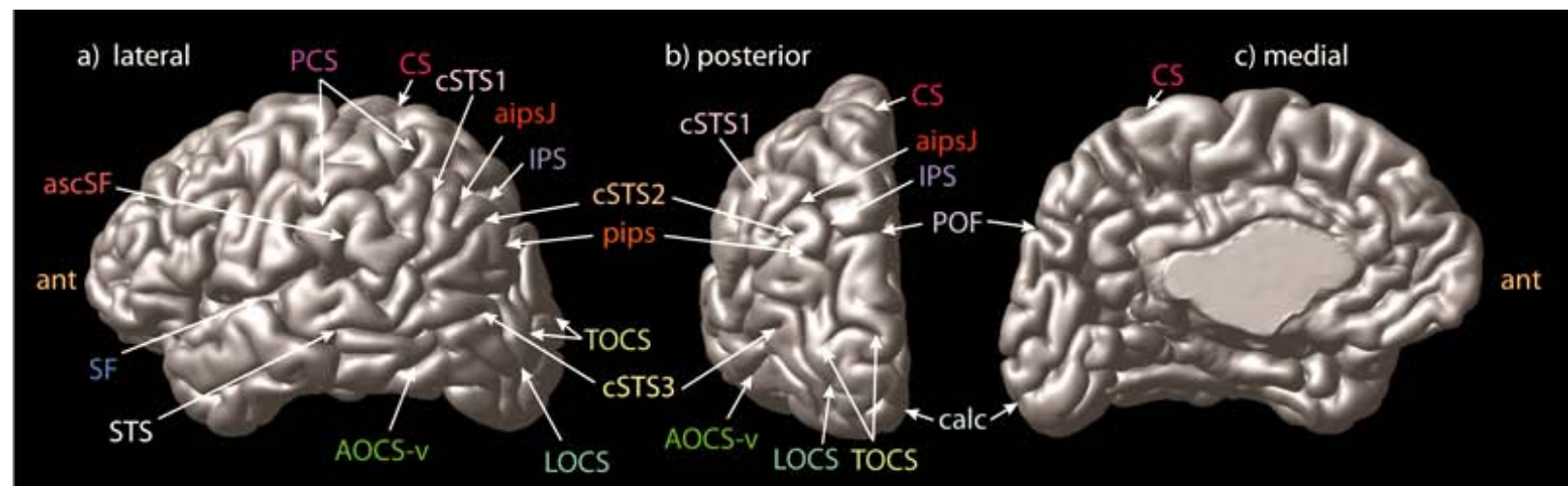

d) medial

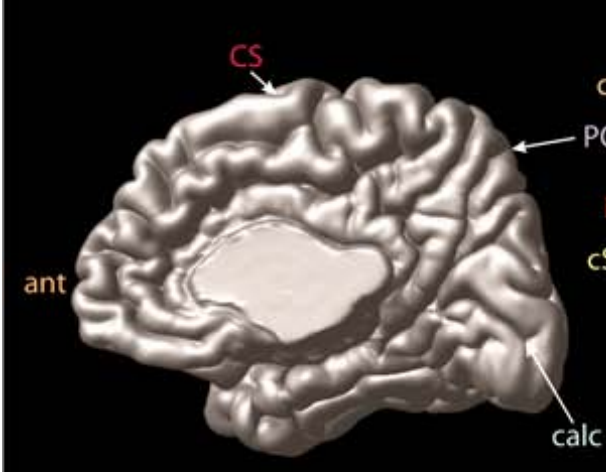

e) posterior

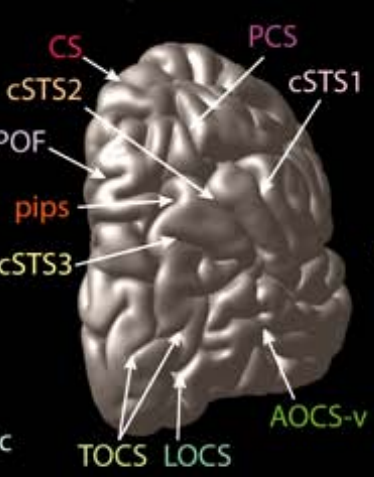

f) lateral

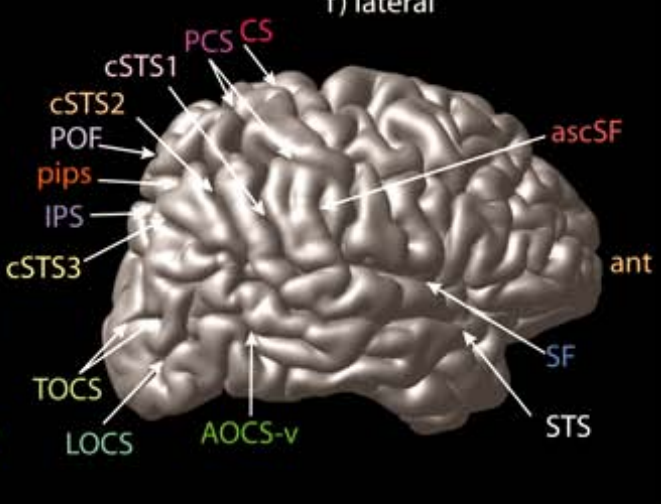

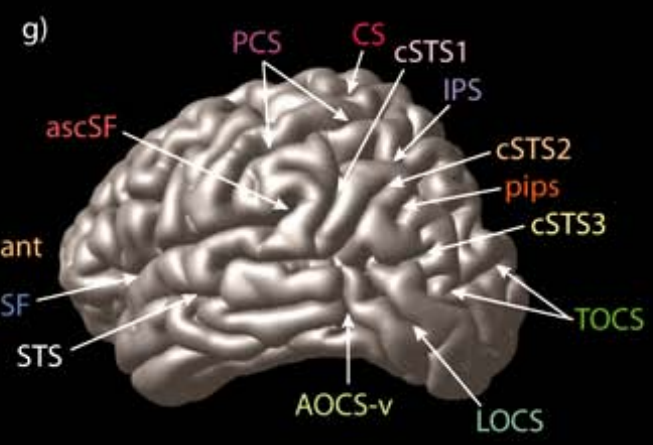

j) $x=-55$

k) $x=-51$

h)

$x=-63$

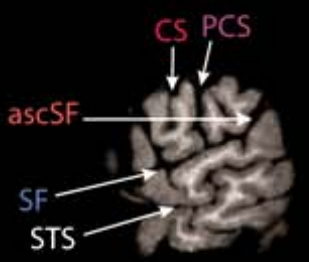

STS $\mathrm{CH}$

i) $x=-59$

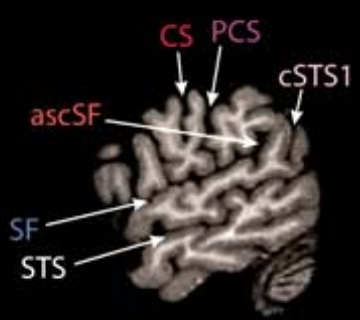

I) $x=-47$ m) $x=-43$

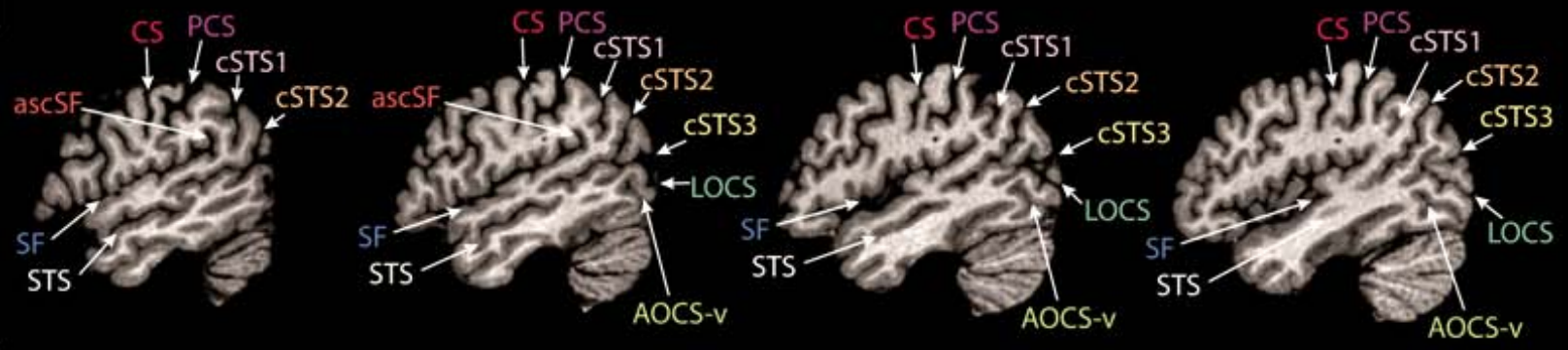


Figure 3.8. (A-F) Lateral, posterior and medial views of $(\mathrm{A}-\mathrm{C})$ the left and $(\mathrm{D}-\mathrm{F})$ the right hemispheres show that the terminal end of the cSTS3 points towards the POF. Note that, in (A, B) the left hemisphere, the cSTS3 is more ventrally located than $(\mathrm{D}, \mathrm{E})$ the right hemisphere. Note also that, following the displacements of CSTS3, the POF in $(B, C)$ the left hemisphere is more ventrally situated than the POF in (E, F) the right hemisphere. (G-M) The temporal lobe segment of the STS appears to join with both the cSTS3 and the cSTS2, according to (G) the 3-D lateral surface view, but (h-m) the sagittal sections show that in this case, in the sulcal depth, the temporal lobe segment of the STS does not join with the cSTS3 but instead joins with the cSTS2, thus highlighting the importance of examining the sulcal depths and going beyond the lateral surface view. ant, anterior; L, left hemisphere; SF, Sylvian fissure; other abbreviations in Figure 3.2 and main list. 


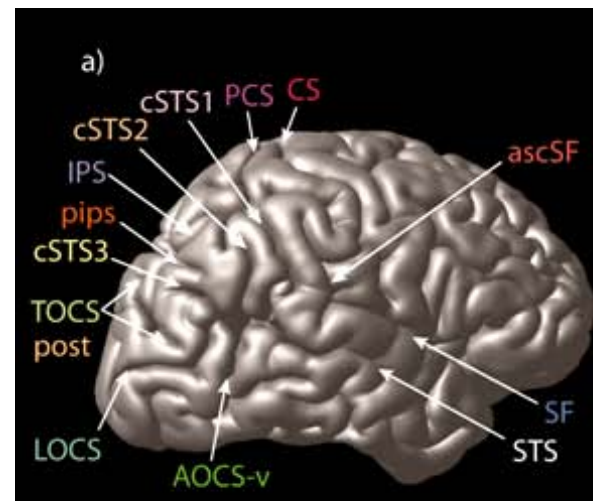

b) $\quad x=49$

c) $\quad x=47$

d)
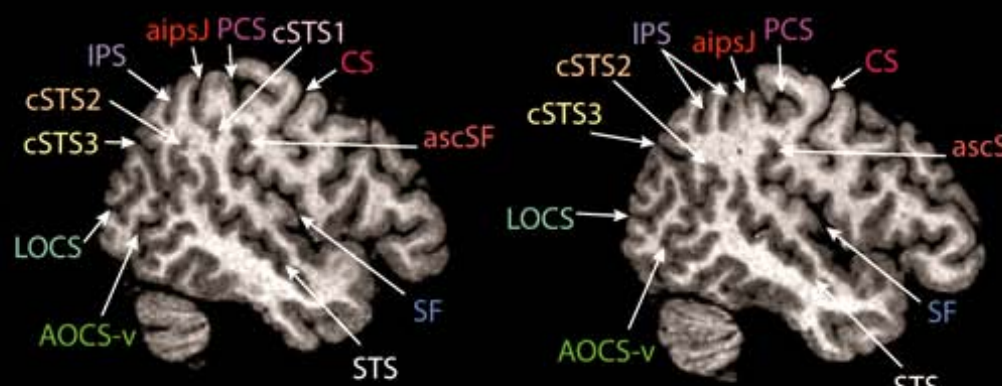

e) $\quad x=43$

f)

STS
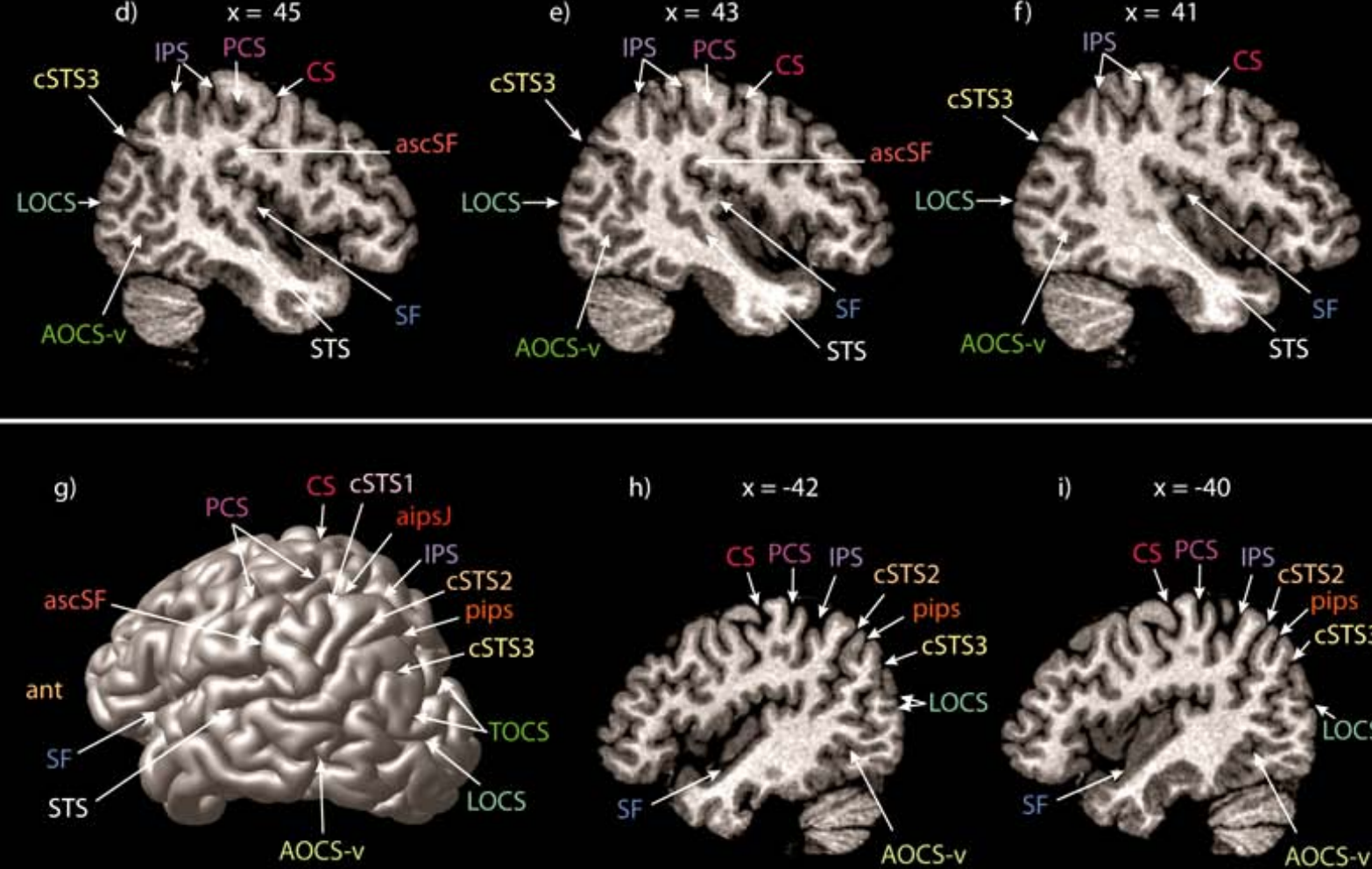

h)

$x=-42$

i) $x=-40$

j)

$x=-38$

k)

$x=-36$

I)

$x=-34$

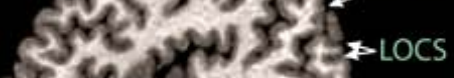
ifist at SF $\frac{7}{2} g^{2}$ AOCS-V

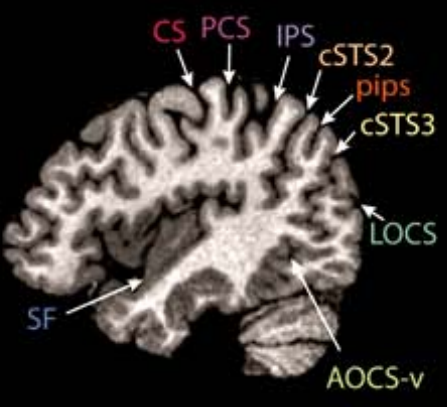

OCS-v

CSCS IPS CSTS2

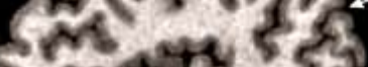

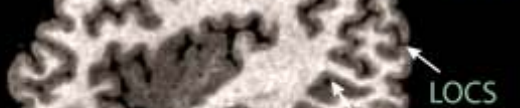

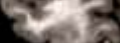

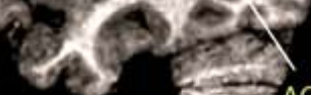

STES AOCS-V

CSPCS IPS

$t l^{1}$ CSTS2

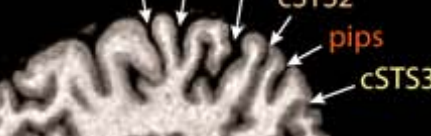

\section{SN} 3 sitis $\sum_{3}$ asur are tors Les 13 . ing

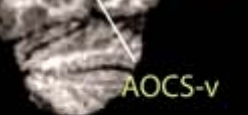

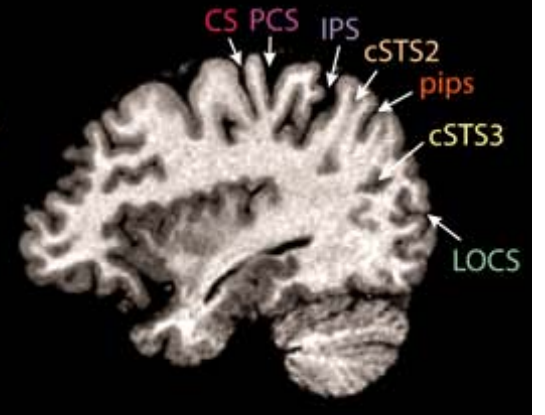


Figure 3.9. (A-F) The dorsal extent of the AOCS-v may reach dorsally to make contact superficially with the cSTS3, according (A) to the 3-D lateral surface view. (B-F) Sagittal sections demonstrate that the two sulci are separated from one another in the sulcal depths. (GL) The LOCS may appear to join the cSTS3, according to (G) the 3-D lateral surface view, but (H-L) the sagittal sections show that, in the sulcal depths, the LOCS is separate from the cSTS3. ant, anterior; L, left hemisphere; SF, Sylvian fissure; other abbreviations in Figure 3.2 and main list. 


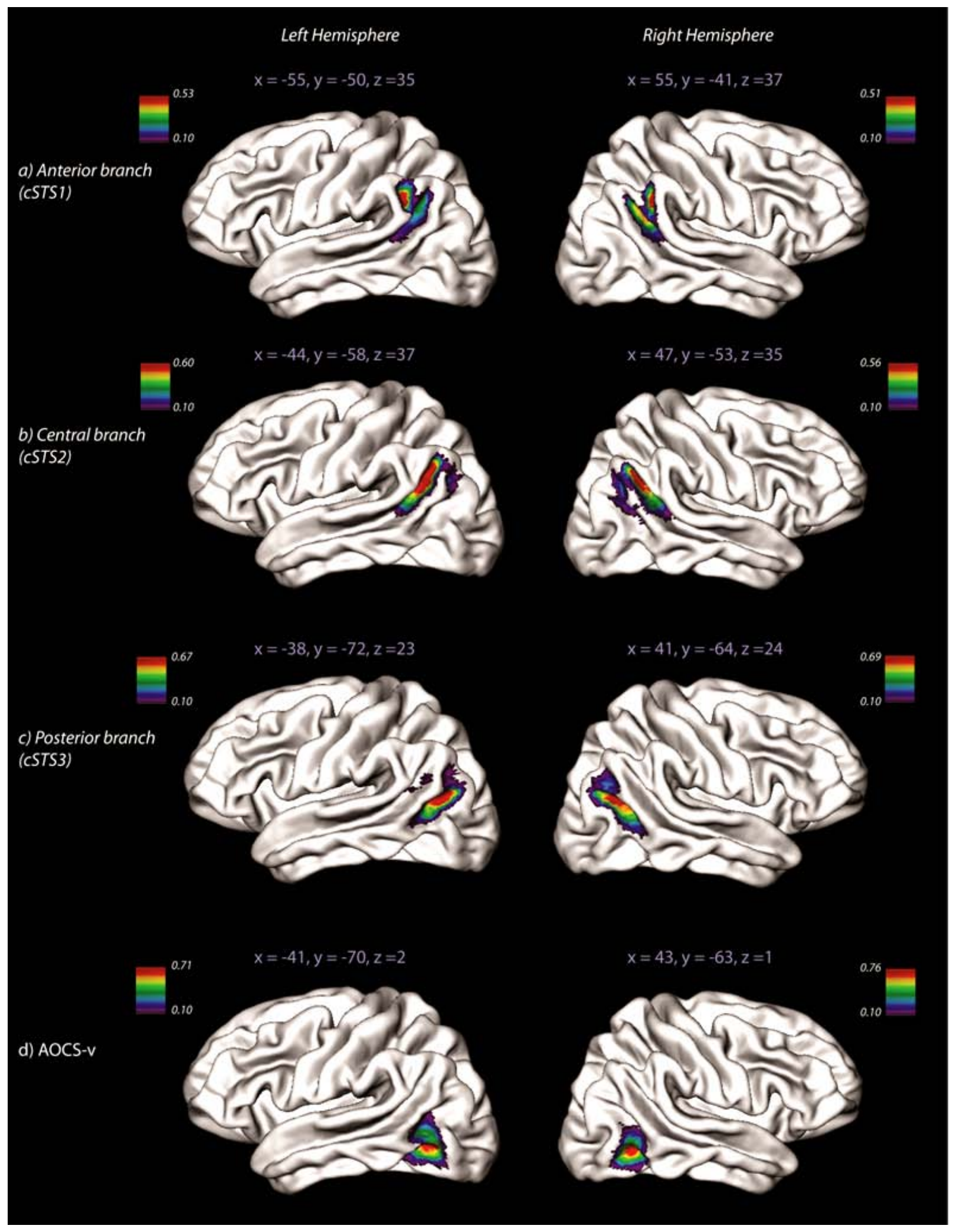


Figure 3.10. The probability maps for each cSTS branch are shown on an average mid-surface of the 45 left hemispheres (left column) and 45 right hemispheres (right column). (A) cSTS1, (B) cSTS2, (C) cSTS3 and (D) AOCS-v. The minimum value for each scale is 0.1 (10\% of the subjects included in this study). The highest probability value varies for the different branches, indicated by the maximum value on each color bar. The $\mathrm{x}, \mathrm{y}, \mathrm{z}$ coordinates reported above each of the brains in (A-D) express the location in MNI standard stereotaxic space with the highest probability of being identified as each of the branches. 


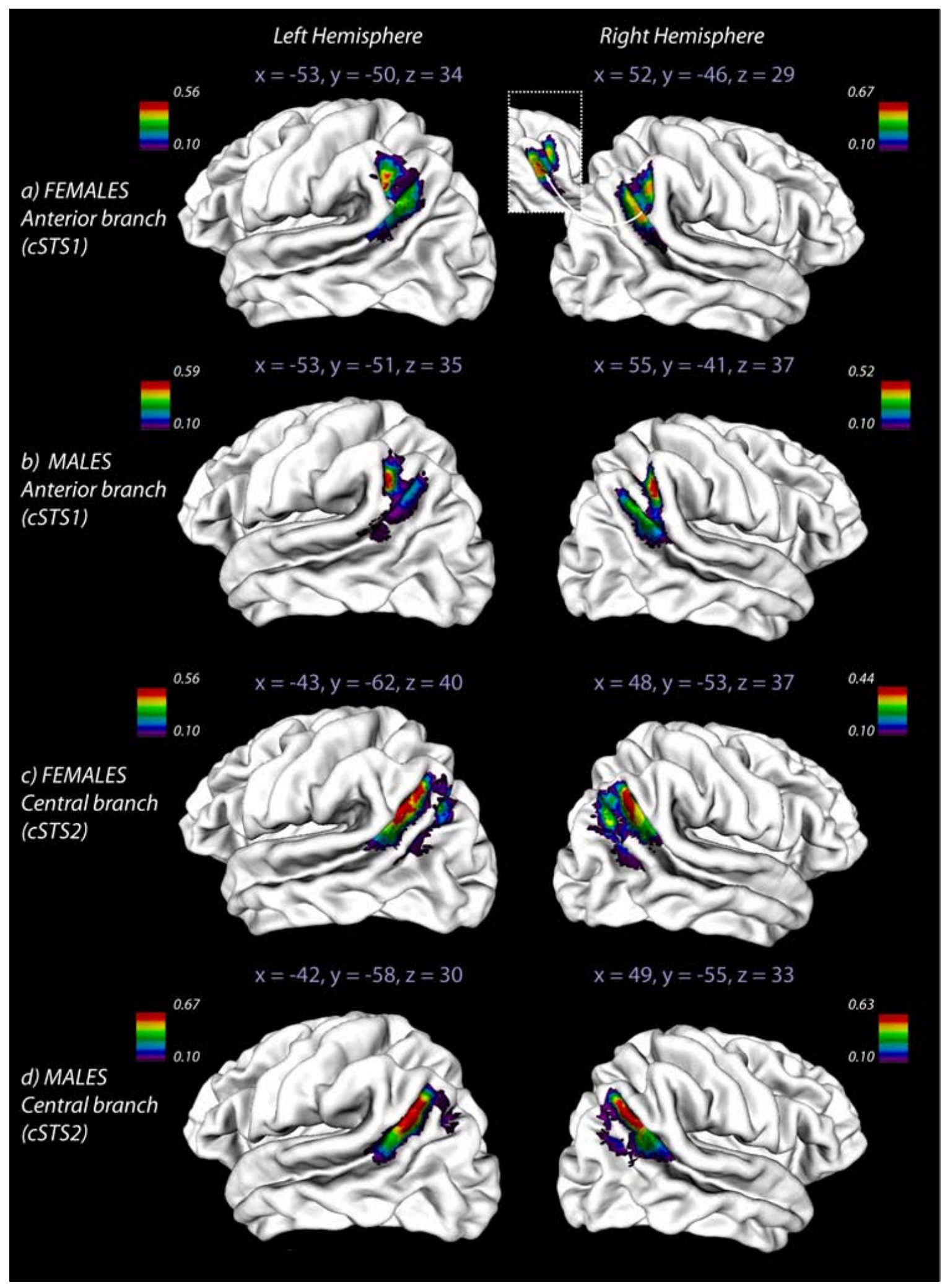


Figure 3.11. Probability maps of cSTS1 and cSTS2 for the left and right hemispheres, presented separately for (A and C) female subjects and for (B and D) male subjects. (A) The probability map of the cSTS1 for female subjects is superimposed on an average registered female midsurface brain and(B) the probability map of the cSTS1 for male subjects is superimposed on an average registered male mid-surface brain. Similarly, (C) the probability map of cSTS2 for female subjects is superimposed on an average registered female mid-surface brain and (D) the probability map of cSTS2 for male subjects is superimposed on an average registered male midsurface brain. The minimum value for each scale is 0.1 (10\% of the subjects included in this study). The highest probability value varies for the different branches, indicated by the maximum value on each color bar. The $\mathrm{x}, \mathrm{y}, \mathrm{z}$ coordinates reported above each of the brains in (A-D) express the location in MNI standard stereotaxic space with the highest probability of being identified as each of the branches. 


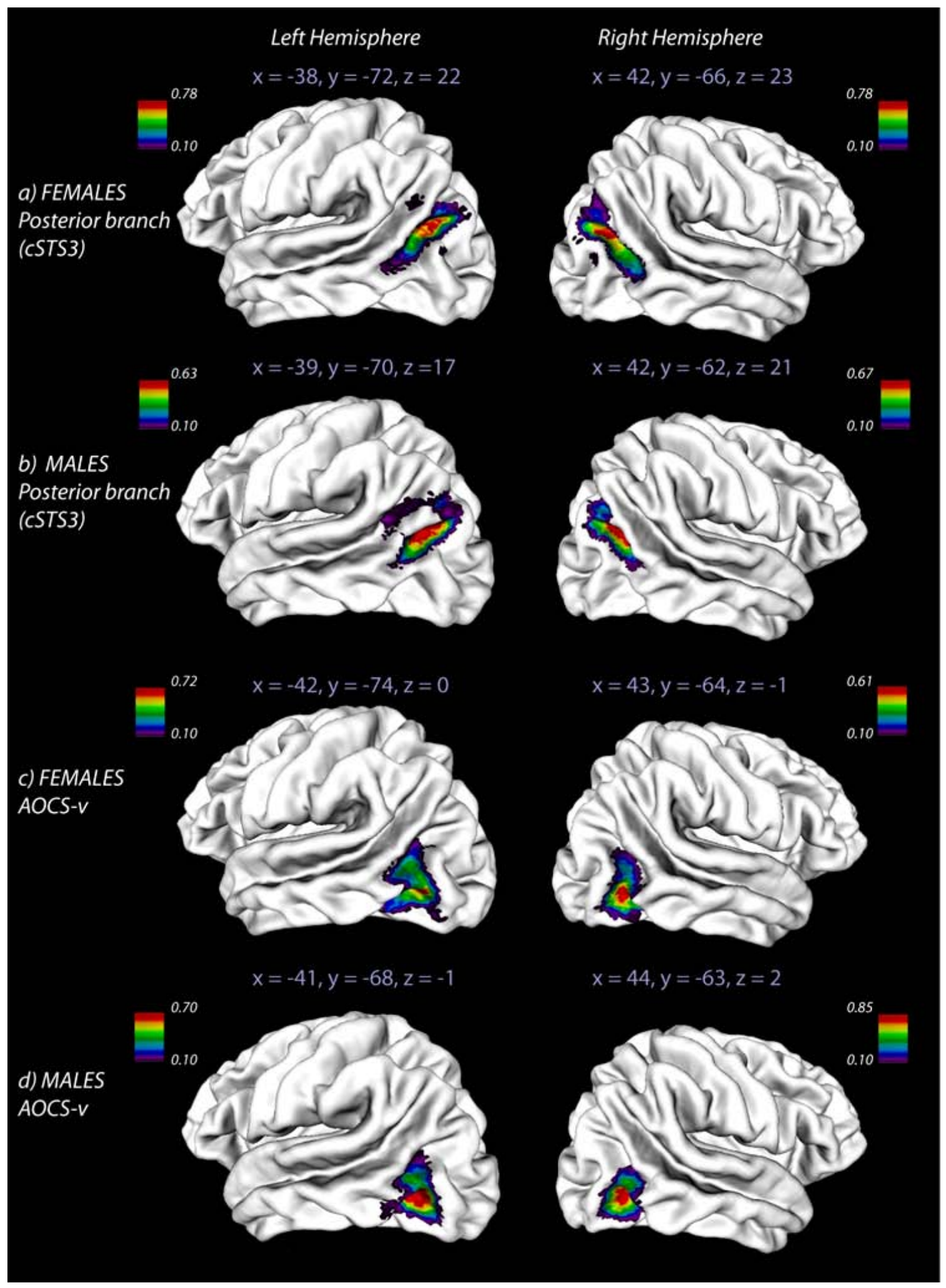


Figure 3.12. Probability maps of cSTS3 and AOCS-v for the left and right hemispheres, presented separately for female subjects and for male subjects. (A) The probability map of the cSTS3 for female subjects is superimposed on an average registered female mid-surface brain and (B) the probability map of the cSTS3 for male subjects is superimposed on an average registered male mid-surface brain. Similarly, (C) the probability maps of AOCS-v for female subjects is superimposed on an average registered female mid-surface brain and (D) the probability maps of AOCS-v for male subjects is superimposed on an average registered male mid-surface brain. The minimum value for each scale is 0.1 (10\% of the subjects included in this study). The highest probability value varies for the different sulci, indicated by the maximum value on each color bar. The $\mathrm{x}, \mathrm{y}, \mathrm{z}$ coordinates reported above each of the brains in (A-D) express the location in MNI standard stereotaxic space (ICBM 152 nonlinear) with the highest probability of being identified as each of the branches. 
a)

$x=-41, y=-69, z=2$

(coordinate from the study by Watson et al., 1993)

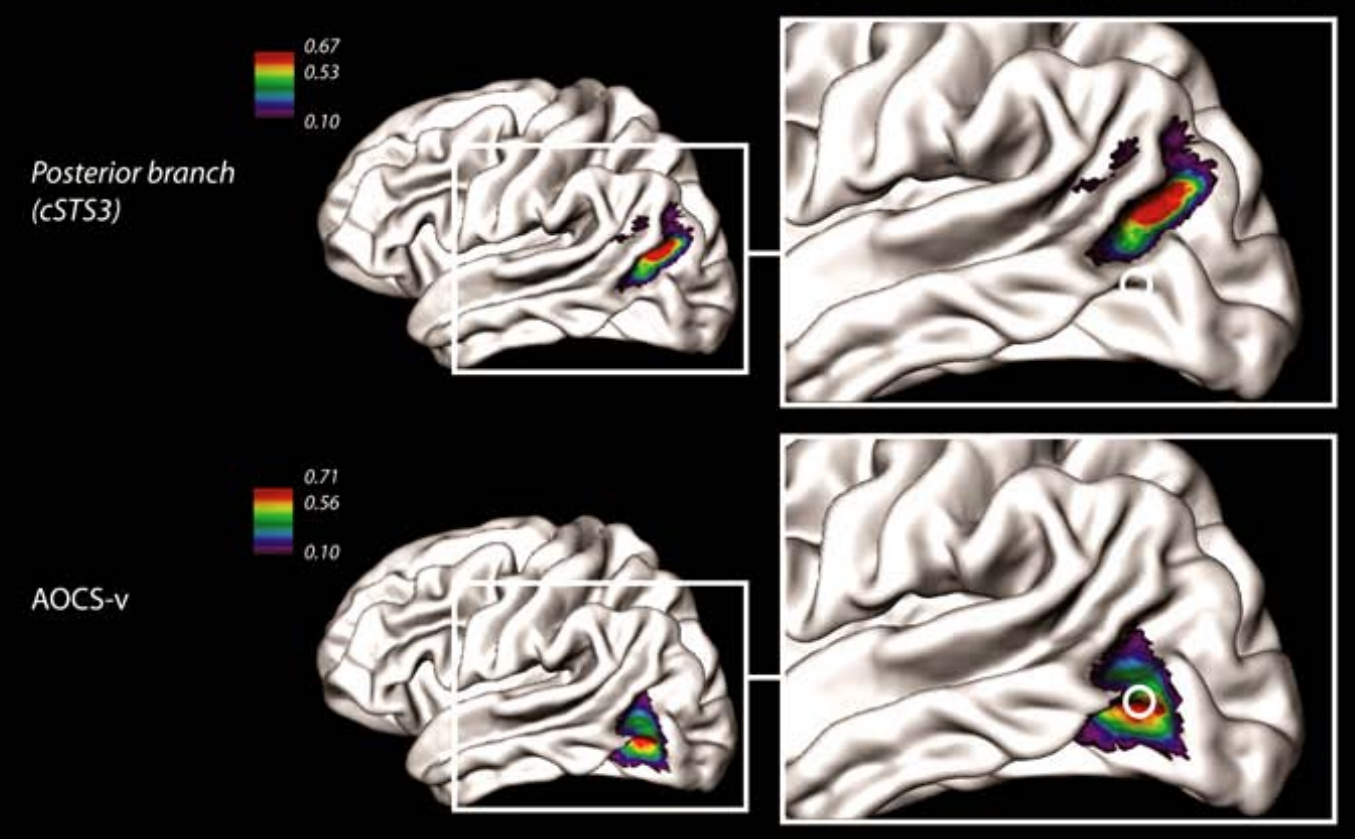

b)

Posterior branch (CSTS3)

$$
0.53
$$$$
x=-51, y=-72, z=10
$$

coordinate from the study by Milakovic et al., 2007)

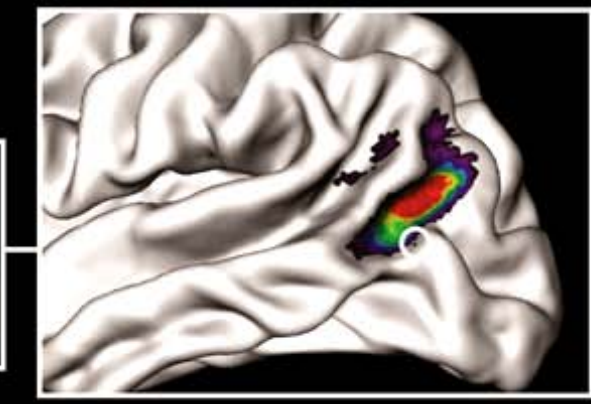

AOCS-V

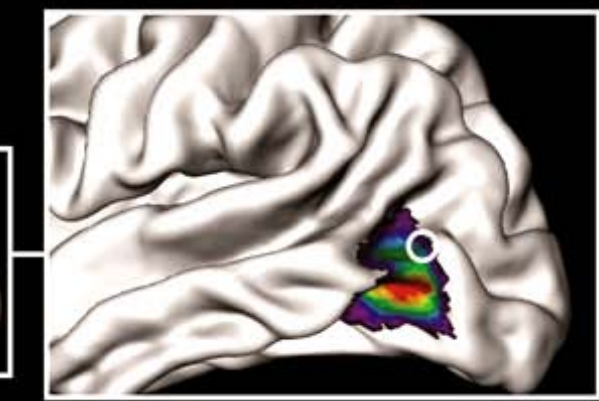


Figure 3.13. The stereotaxic coordinates of motion are $\$$ Tas determined by past neuroimaging studies in humans is presented in relation to the probability maps of the cSTS3 and of the AOCS-v from the present study. (A) The standard stereotaxic MNI coordinates (reported above the magnified brain area) from the neuroimaging study of area MT/v5 in the human brain by Watson et al. (1993) entered into the probability maps from the present study (from Figure 3.10) show its location in relation to the location of cSTS3 and AOCS-v (enlarged area). The white circle on the lateral surface surrounds the vertex of the average mid-surface brain that corresponds to the MNI coordinates from the functional neuroimaging study. (B) The standard stereotaxic MNI coordinates (reported above the magnified brain area) from the cytoarchitectonic study by Malikovic et al. (2007) entered into the probability maps from the present study (from Figure 3.10) to show the location of the putative cytoarchitectonic MT5 area in relation to the location of cSTS3 and AOCS-v (enlarged area). The white circle on the lateral surface surrounds the vertex of the average mid-surface brain that corresponds to the MNI coordinates from the cytoarchitectonic study. The color bar in (A) and (B) indicates the range in probability that a given set of coordinates is identified as a particular sulcus. The minimum value for each scale is 0.1 ( $10 \%$ of the subjects included in this study). The highest probability value varies for the different branches, indicated by the maximum value on each color bar. 